

Riehl, S. and Steinmann, P. (2017) On structural shape optimization using an embedding domain discretization technique. International Journal for Numerical Methods in Engineering, 109(9), pp. 1315-1343. (doi:10.1002/nme.5326).

There may be differences between this version and the published version. You are advised to consult the publisher's version if you wish to cite from it.

This is the peer reviewed version of the following article: Riehl, S. and Steinmann, P. (2017) On structural shape optimization using an embedding domain discretization technique. International Journal for Numerical Methods in Engineering, 109(9), pp. 1315-1343, which has been published in final form at 10.1002/nme.5326. This article may be used for non-commercial purposes in accordance with Wiley Terms and Conditions for Self-Archiving.

http://eprints.gla.ac.uk/185715/

Deposited on: 14 June 2019

Enlighten - Research publications by members of the University of Glasgow http://eprints.gla.ac.uk 


\title{
On structural shape optimization using an embedding domain discretization technique
}

\author{
Stefan Riehl ${ }^{\mathrm{a}}$, Paul Steinmann ${ }^{\mathrm{a}, *}$ \\ ${ }^{a}$ Chair of Applied Mechanics, Friedrich-Alexander University Erlangen-Nürnberg, Egerlandstr. 5, \\ 91058 Erlangen, Germany
}

\begin{abstract}
This contribution presents a novel approach to structural shape optimization that relies on an embedding domain discretization technique. The evolving shape design is embedded within a uniform finite element background mesh which is then used for the solution of the physical state problem throughout the course of the optimization. We consider a boundary tracking procedure based on adaptive mesh refinement to separate between interior elements, exterior elements, and elements intersected by the physical domain boundary. A selective domain integration procedure is employed to account for the geometric mismatch between the uniform embedding domain discretization and the evolving structural component. Thereby, we avoid the need to provide a finite element mesh that conforms to the structural component for every design iteration, as it is the case for a standard Lagrangian approach to structural shape optimization. Still, we adopt an explicit shape parametrization that allows for a direct manipulation of boundary vertices for the design evolution process. In order to avoid irregular and impracticable design updates, we consider a geometric regularization technique to render feasible descent directions for the course of the optimization.
\end{abstract}

Keywords: finite element, embedding domain, shape optimization, design sensitivity analysis

\section{Introduction}

Along with the advent of the finite element method in the engineering community in the 1970s, the field of structural shape optimization has emerged as a promising area of application. Early attempts in this field of research, however, revealed severe drawbacks when altering a structural design component through a direct manipulation of nodal point coordinates in its finite element representation $[1,2,3]$. Not only did one suffer from undesired mesh dependency effects, such as a varying structural response when perturbing interior nodal points, but also struggled with irregular and impracticable design updates at the domain boundary.

Even more, in order to represent comparatively large shape changes using one and the same finite element mesh, one needs to accept a highly non-uniform interior mesh distribution at later design iterations. And in some cases the premature termination of the domain update procedure

*Corresponding author: Phone: $+49(0) 913185$ 28501, Fax: $+49(0) 91318528503$

Email address: paul.steinmann@ltm.uni-erlangen.de (Paul Steinmann) 
is unavoidable due to severe element distortions.

Two main considerations have evolved to bypass these conceptual shortcomings. On the one hand, a separate geometry model can be employed to govern the design update procedure $[4,5$, $6,7]$. By this mechanism, the inconvenient direct manipulation of nodal point coordinates within the corresponding finite element representation is avoided. Based on the respective parametrization of the geometry model ${ }^{1}$, the admissible design space for the course of optimization is limited, and hence the development of impracticable zig-zag patterns at the domain boundary is overcome. In fact, these undesired patterns can no longer be represented by any combination of design variables and are effectively excluded from the available design space for optimization.

On the other hand, the initial finite element analysis model may be updated during the course of the optimization not only to adhere to the varying boundary of the geometry model, but also to improve element mesh quality or the accuracy of the numerical analysis results $[8,9,10,11]$. This may for instance take the form of geometric mesh smoothing operations, and goal-oriented or error-controlled adaptive mesh refinement. However, these mechanisms may still encounter difficulties when it comes to large shape changes, or even topological changes throughout the course of the optimization, which then necessitates the use of an elaborate, computationally expensive re-meshing algorithm that is conducted in turn with the domain update procedure.

From this perspective, so-called embedding (or fictitious) domain discretization techniques seem an ideal supplement and a natural choice for the application in structural shape optimization $[12,13,14,15,16,17]$. For these methods, the main idea is to extend the structural analysis problem to a regularly-shaped auxiliary domain that encloses the physical domain boundary. Then, for the approximate numerical solution of the structural analysis problem, it suffices to provide a uniform discretization for the auxiliary domain, rather than a finite element mesh that conforms the arbitrary-shaped structural component itself. Further, when considering domain variations of the structural component, its material points are not attached to finite element nodal points but rather move through the stationary finite element mesh of the embedding domain such that no mesh distortion is observed.

However, these apparent advantages do not come along without numerical challenges. In fact, the computational effort is shifted from the use of reliable meshing algorithms towards elaborate numerical integration schemes. This is to account for the geometric mismatch between the physical domain boundary and its non-conforming finite element representation within the auxiliary domain setting. All elements that are intersected by the boundary of the structural component are subject to a selective domain integration procedure to distinguish the respective element area fractions interior and exterior to the physical domain boundary. This selective domain integration procedure is crucial to the numerical accuracy that is attributed to the embedding domain analysis results, and a number of different approaches have been investigated in the literature. A first approach, common to applications in topology optimization $[18,19]$, is to homogenize (or distribute evenly) the material characteristics within intersected elements based on the respective interior element area fraction. Thereby, intersected elements are taken into account by use of a standard quadrature rule, only to scale down all integral contributions by the interior element area fraction subsequently. This approach is computationally efficient but is also known to provide comparatively low accuracy for the embedding domain structural analysis [20,21].

A more selective approach to track the physical boundary within the embedding finite element

\footnotetext{
${ }^{1} \mathrm{~A}$ basic parametrization of the geometry model may for instance comprise a set of straight lines, different radii, or a set of interconnected spline curves. Also, more elaborate geometry descriptions based on Bezier curves or NURBS parametrizations may be taken into account.
} 
domain is to provide for all intersected elements a greater number of integration points as is necessary for the exact integration of the intrinsic finite element polynomial space. Each integration point is then checked to be interior or exterior to the physical domain boundary [22]. Based on this classification, the integral contribution from each integration point is either taken into account, or neglected for the evaluation of the respective element domain integral. However, even for a large number of integration points, the sharp intra-element material discontinuity cannot be represented exactly by this procedure. A conceptually related approach is employed in the finite cell framework $[23,24,25]$. Therein, intersected elements are subject to a selective domain integration employing hierarchical tree data structures, a computationally efficient procedure on the basis of nested element subdivisions.

Still, the highest level of accuracy for the embedding domain structural analysis is achieved only when an exact integration of intersected elements is taken into account. One approach to meet this requirement is to partly loosen the uniform mesh principle in the embedding domain setting. More specifically, one aims to re-orient a layer of finite elements within the embedding domain discretization such as to conform the physical domain boundary [26, 27, 28, 29, 30]. Thereby, one enables the use of a standard quadrature rule for the exact domain integration. On the contrary, an exact domain integration can also be realized by the use of a separate intra-element sub-triangulation that is used for integration purposes only $[12,31]$.

The present contribution is concerned with the formulation and implementation of a method for structural shape optimization in an embedding domain setting. The method relies on an explicit geometry description and all investigations are limited to a linear elastic regime in twodimensional space. An adjoint formulation is used for the derivation of design sensitivities in the continuous setting, in which we follow the material derivative concept from continuum mechanics. We consider different numerical examples in which we assess the applicability of different selective domain integration procedures for the use in structural shape optimization. This includes the evaluation of their computational efficiency, ease of implementation, and the expected increase in complexity when considering an extension to a three-dimensional setting.

As opposed to existing contributions in the literature that rely on an explicit but parametric design description [12, 13, 14], we allow for a completely free modification of the evolving shape design by a direct and independent manipulation of all contour vertices available. This is to allow for a large design space to choose optimal solutions from. However, due to the increasing number of design variables and the independent movement of all vertices we still need to counteract the above-mentioned development of impracticable zig-zag patterns at the evolving contour curve.

We address this issue by two mechanisms. First, we consider a hierarchic design description in which we use a relatively coarse contour curve with few design variables to allow for the largest and most characteristic design changes at early design stages. At later design iterations, the contour curve is then provided with additional vertices to allow for the formation of finer scale details within the optimal shape design. As a second mechanism, we consider a descent algorithm for the update process in which we do not rely exclusively on the steepest descent direction obtained from shape sensitivity analysis, but rather a feasible descent direction that results in smoother updates of the contour curve. Thereby, the feasible descent direction comprises a linear combination of the steepest descent direction as well as a local vertex averaging that moves every vertex towards the central point being defined by its adjacent vertices.

As opposed to existing contributions in the literature that rely on an implicit design parametrization $[15,16,19,30]$, typically a level-set function, the present method does not require the solution of an additional boundary value problem (e.g. the Hamilton-Jacobi equation) to obtain an overall design velocity field to update the evolving shape design, but allows for a direct and inde- 
pendent manipulation of all contour vertices. This facilitates the identification and consideration of specific geometric features for the course of the optimization through coupling a subset of the contour vertices, a demand that may arise from certain geometric and manufacturing constraints [32]. One may also think of pre-defined geometric features as part of the optimization process, for instance inclusions of fixed size and orientation but variable positioning, or a fixed number of holes of varying radius. In this perspective, the inherent versatility of the implicit approach to allow for splitting and merging operations of existing holes may not be practicable in certain engineering or industrial close-to-production design scenarios.

In contrast to $[33,34]$, the present explicit approach involves a strict and straightforward separation of the evolving shape design and the embedding domain mesh since the former is stand-alone and not obtained indirectly from the nodal points of the unstructured analysis mesh. Thereby, refinements of the analysis mesh do not change the parametrization and thus the geometric versatility of the contour curve, and refinements of the contour curve do not alter the result (or accuracy) of the analysis mesh. In fact, the analysis mesh may be equipped with an independent a posteriori FE solution-based refinement scheme to render equally accurate results throughout the course of the optimization.

\section{Embedding domain technique - continuum formulation}

We consider an elastic body B occupying a bounded open domain $\mathcal{B} \subset \mathcal{R}^{2}$ that comprises of continuum points $\mathbf{X}$ and to which we attribute homogeneous and isotropic material characteristics. More specifically, we assume that the structural response of the elastic body due to external loading is determined by a linear Hookean material parametrization using Lamé parameters $\lambda$ and $\mu$, such that the Cauchy stress tensor is in the format

$$
\sigma(\boldsymbol{\epsilon})=\lambda[\operatorname{tr} \boldsymbol{\epsilon}] \mathbf{I}+2 \mu \boldsymbol{\epsilon}
$$

where $\boldsymbol{\epsilon}=\nabla^{\mathrm{sym}} \mathbf{u}$ using the symmetric gradient operator $\nabla^{\mathrm{sym}}\{\bullet\}=1 /{ }_{2}[\nabla\{\bullet\}]+{ }_{1}^{1}{ }_{2}[\nabla\{\bullet\}]^{T}$, and $\mathbf{I}$ denotes the second-order identity tensor.

The boundary $\Gamma$ of $\mathcal{B}$ consists of two disjoint portions $\Gamma^{N}$ and $\Gamma^{D}$, such that

$$
\Gamma^{N} \cup \Gamma^{D}=\Gamma, \Gamma^{N} \cap \Gamma^{D}=\emptyset .
$$

The body $\mathrm{B}$ is subject to volume forces $\mathbf{b}$ being specified per unit volume in $\mathcal{B}$ and traction forces $\mathbf{t}$ acting on $\Gamma^{N}$, whereas $\Gamma^{D}$ is equipped with prescribed displacements $\overline{\mathbf{u}}$.

Spatial equilibrium is then obtained for the displacement function $\mathbf{u}$ that fulfils the Euler-Lagrange equations in the format

$$
\begin{array}{ll}
\operatorname{div} \boldsymbol{\sigma}+\mathbf{b}=\mathbf{0} & \text { in } \mathcal{B} \\
\boldsymbol{\sigma} \cdot \mathbf{n}=\mathbf{t} & \text { on } \Gamma^{N} \\
\mathbf{u}=\overline{\mathbf{u}} & \text { on } \Gamma^{D},
\end{array}
$$

where $\mathbf{n}$ denotes the outward unit normal to $\Gamma^{N}$.

In order to establish a variational formulation, we consider an admissible virtual displacement function $\delta \mathbf{u}^{2}$ and integrate the localized force balance in Eq. (3) over $\mathcal{B}$ to render the principle of virtual work

$$
\int_{\mathcal{B}} \boldsymbol{\sigma}: \delta \boldsymbol{\epsilon} \mathrm{dA}=\int_{\mathcal{B}} \mathbf{b} \cdot \delta \mathbf{u} \mathrm{dA}+\int_{\Gamma^{N}} \mathbf{t} \cdot \delta \mathbf{u} \mathrm{dS}
$$

\footnotetext{
${ }^{2} \delta \mathbf{u}=\mathbf{0}$ on $\Gamma^{D}$.
} 


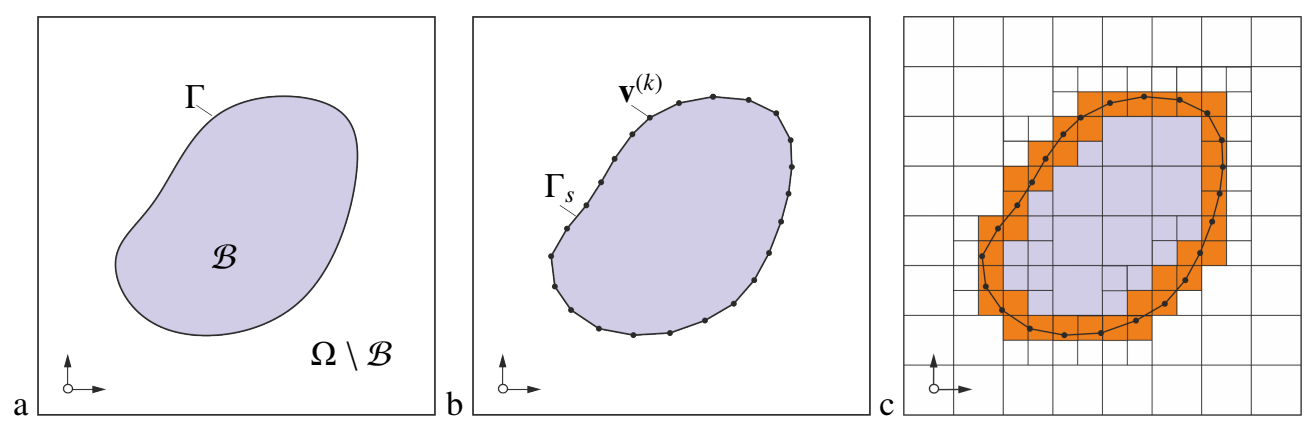

Figure 1: Conceptual design and finite element modelling of the embedding domain approach. a An elastic body B occupying a bounded open set $\mathcal{B} \subset \mathcal{R}^{2}$ with boundary $\Gamma$ is embedded within a regularly-shaped domain $\Omega$. b For simplification, we consider a polygonal representation $\Gamma^{h}$ of the physical boundary $\Gamma$ in the sequel. $\mathbf{c}$ Upon discretization of the embedding domain $\Omega$, three sets of finite elements are identified based on their relative positioning against $\Gamma^{h}$ : exterior (white), interior (blue), and elements intersected by $\Gamma^{h}$ (orange).

where $\delta \boldsymbol{\epsilon}=\nabla^{\mathrm{sym}} \delta \mathbf{u}$.

At this point, let us consider $\mathcal{B}$ to be a subset of a regularly-shaped embedding domain $\Omega$, cf. Fig. 1a. Then, introducing a characteristic function $\chi$ such that

$$
\chi(\mathbf{X})= \begin{cases}1 & \text { if } \mathbf{X} \in \mathcal{B} \\ 0 & \text { if } \mathbf{X} \in \Omega \backslash \mathcal{B},\end{cases}
$$

the virtual work statement in Eq. (4) is equally expressed through

$$
\int_{\Omega} \chi \boldsymbol{\sigma}: \delta \boldsymbol{\epsilon} \mathrm{dA}=\int_{\Omega} \chi \mathbf{b} \cdot \delta \mathbf{u} \mathrm{dA}+\int_{\Gamma^{N}} \mathbf{t} \cdot \delta \mathbf{u} \mathrm{dS},
$$

where we consider the extension of the primary solution variable $\mathbf{u}=\mathbf{0}$ for $\mathbf{X} \in \Omega \backslash \mathcal{B}$.

The benefit of the virtual work formulation in Eq. (6) as opposed to the unaltered formulation in Eq. (4) is a change of the domain for integration, which circumvents the need to provide a boundary conforming finite element discretization $\mathcal{B}^{h}$ of $\mathcal{B}$.

For ease of notation, let us formally refer to the virtual work expression (6) via

$$
W_{\chi}(\mathbf{u}, \delta \mathbf{u})=a_{\chi}(\mathbf{u}, \delta \mathbf{u})-l_{\chi}(\delta \mathbf{u})=0,
$$

where $a_{\chi}$ and $l_{\chi}$ denote the (energy) bilinear and the (load) linear form, respectively

$$
\begin{gathered}
a_{\chi}(\mathbf{u}, \delta \mathbf{u})=\int_{\Omega} \chi \boldsymbol{\sigma}: \delta \boldsymbol{\epsilon} \mathrm{dA}, \\
l_{\chi}(\delta \mathbf{u})=\int_{\Omega} \chi \mathbf{b} \cdot \delta \mathbf{u} \mathrm{dA}+\int_{\Gamma^{N}} \mathbf{t} \cdot \delta \mathbf{u} \mathrm{dS} .
\end{gathered}
$$

\section{Finite element modelling}

For the approximate solution of the virtual work statement in Eq. (7) within a finite element setting, we consider a collection of $n_{e}$ (regular square) finite elements $\Omega_{e}$ to establish a discrete domain representation $\Omega^{h}$ of $\Omega$

$$
\Omega \approx \Omega^{h}=\bigcup_{e=1}^{n_{e}} \Omega_{e} .
$$


The corresponding elementwise placement $\mathbf{X}_{e}$ and the elementwise displacement function $\mathbf{u}_{e}$ are given in terms of the corresponding nodal point values $\mathbf{X}^{(i)}, \mathbf{u}^{(i)}$ and polynomial shape functions $N^{(i)}(\boldsymbol{\xi})$ parametrized by isoparametric coordinates $\boldsymbol{\xi} \in[-1,1]^{2}$

$$
\mathbf{X}_{e}(\boldsymbol{\xi})=\sum_{i=1}^{n_{e n}} N^{(i)}(\boldsymbol{\xi}) \mathbf{X}^{(i)}, \quad \mathbf{u}_{e}(\boldsymbol{\xi})=\sum_{i=1}^{n_{e n}} N^{(i)}(\boldsymbol{\xi}) \mathbf{u}^{(i)}
$$

where $n_{e n}$ denotes the number of nodal points per element.

In the sequel, we allow for a geometric simplification in which we consider $\mathcal{B}$ to be bound by a polygonal approximation $\Gamma^{h}$ of $\Gamma$ using piecewise linear interpolation between its $n_{v}$ vertices $\mathbf{v}^{(k)}$, cf. Fig. 1b. Further, we denote by $\Gamma_{s}$ the respective adjoining $n_{s}$ line segments ${ }^{3}$ such that $\Gamma^{h}=\bigcup_{s=1}^{n_{s}} \Gamma_{s}$. As a result, we distinguish three sets of finite elements to which individual elements $\Omega_{e}$ are assigned based on their relative positioning as compared to $\Gamma^{h}$, cf. Fig. 1c. Specifically, we denote by $\{\Omega\}^{\text {int }}$ the set that comprises all $\Omega_{e}$ interior $\Gamma^{h}$, and we denote by $\{\Omega\}^{\text {ext }}$ the set that comprises all $\Omega_{e}$ exterior $\Gamma^{h}$. The remaining elements $\Omega_{e}$ are intersected by $\Gamma^{h}$ and are identified within $\{\Omega\}^{\text {bnd }}$.

Moreover, we do not rely on a uniform discretization $\Omega^{h}$ for the embedding domain, but rather aim to resolve the physical boundary $\Gamma^{h}$ more closely. To this extent, starting from a coarselevel discretization $\Omega^{h}$, we invoke a tracking mechanism for the domain boundary $\Gamma^{h}$ based on adaptive (or hierarchical) mesh refinement. Thereby, we seek to narrow the layer of elements identified within $\{\Omega\}^{\text {bnd }}$ until a user-specified threshold value $\gamma$ that is relating the area of $\{\Omega\}^{\text {bnd }}$ and the length of $\Gamma^{h}$ is met

$$
\text { area }\left(\{\Omega\}^{\text {bnd }}\right) / \text { length }\left(\Gamma^{h}\right) \leq \gamma .
$$

As a consequence of this tracking procedure, hanging node (or multi-point) constraints are to be considered between neighbouring finite elements showing different levels of refinement, cf. Fig. 2.

In order to account for the remaining geometric mismatch between the physical boundary $\Gamma^{h}$ and its embedding domain approximation via $\{\Omega\}^{\text {bnd }}$, we consider two distinct approaches.

First, within the staircase scheme, we rely on an oversupply of integration points to allow for a more detailed intra-element consideration of $\Gamma^{h}$ within $\{\Omega\}^{\text {bnd }}$. Thereby, considering all domain integrals involving elements within $\{\Omega\}^{\text {bnd }}$, each integration point is checked to be interior or exterior to $\Gamma^{h}$. A detailed account on the specifics of this approach is given in Sec. 3.1.

By contrast, following the reorientation scheme, we invoke a rearrangement procedure for all nodal points being adjacent to elements within $\{\Omega\}^{\text {bnd }}$ in order to obtain a (nearly) conforming boundary layer within $\Omega^{h}$, which then enables the use of a standard numerical integration procedure. The specifics of this approach are outlined in Sec. 3.2.

\subsection{Staircase scheme}

Upon discretization of the virtual work statement in Eq. (7), we obtain a matrix system of equations for the finite element solution vector $\mathbf{u}^{h}$ in the format

$$
\mathbf{K} \mathbf{u}^{h}=\mathbf{f},
$$

\footnotetext{
${ }^{3}$ For closed polygons, we obtain $n_{s} \equiv n_{v}$.
} 

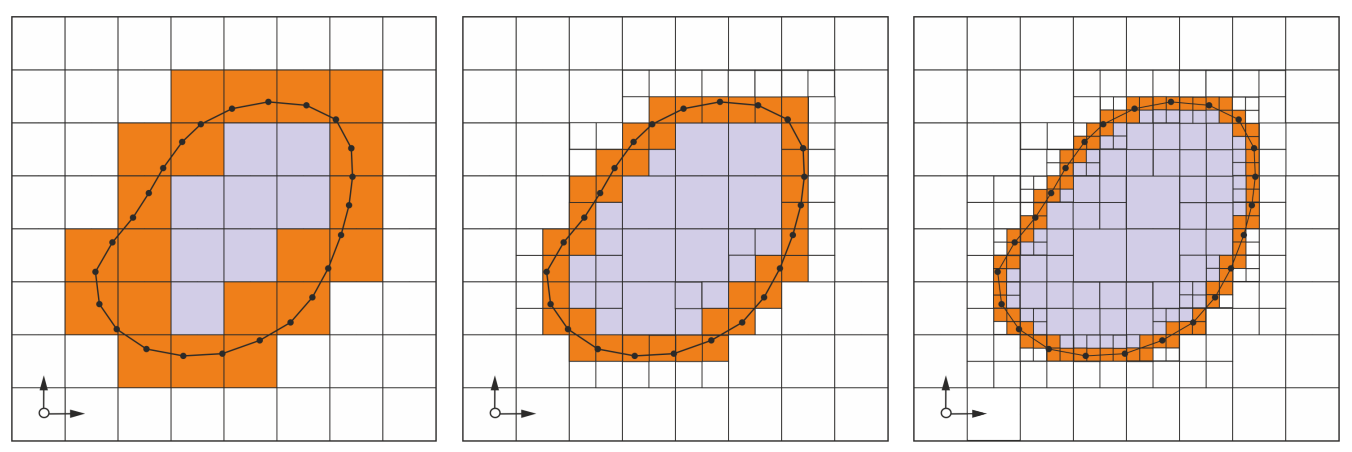

Figure 2: Embedding domain tracking mechanism via adaptive (or hierarchical) mesh refinement. Elements within $\{\Omega\}^{\text {bnd }}$ are marked for refinement until a user-specified criterion $\gamma$ that is relating the respective extent of $\{\Omega\}^{\text {bnd }}$ and $\Gamma^{h}$ is met, cf. Eq. (11).

where $\mathbf{K}$ and $\mathbf{f}$ denote the system stiffness matrix and the external load vector, respectively. The system stiffness matrix $\mathbf{K}$ consists of dual nodal point contributions of the type

$$
\mathbf{K}^{(I J)}=\boldsymbol{A}_{e=1}^{n_{e}} \int_{\Omega_{e}} \chi \nabla N^{(i)} \cdot \mathbb{E} \cdot \nabla N^{(j)} \mathrm{dA},
$$

where capital letters $(I)$ and $(J)$ are used to denote global (or overall) node numbering and small letters $(i)$ and $(j)$ are used to identify local (or elementwise) node numbers. Consequently, the assembly operator ${ }^{4}$ A spans over all elements $\Omega_{e}$ for which $(I) \in \Omega_{e}$ and $(J) \in \Omega_{e}$. The fourthorder elasticity tensor $\mathbb{E}$ in Eq. (13) is expressed in terms of the Lamé parameters

$$
\mathbb{E}=\lambda \mathbf{I} \otimes \mathbf{I}+\mu \mathbf{I} \otimes \mathbf{I}+\mu \mathbf{I} \bar{\otimes} \mathbf{I}
$$

where $\otimes$ and $\bar{\otimes}$ denote non-standard ${ }^{5}$ dyadic products.

The characteristic function $\chi$ in Eq. (13) equals zero for all domain integrals considering elements within $\{\Omega\}^{\mathrm{ext}}$. Hence, no degrees of freedom are assigned to $\{\Omega\}^{\mathrm{ext}}$ and we can exclude the respective elements from the assembly routine. By contrast, since $\chi=1$ for all elements within $\{\Omega\}^{\text {int }}$, a standard numerical integration procedure is used to establish the respective single element contributions in Eq. (13). Therefore, it remains to determine the integral contributions considering all elements within $\{\Omega\}^{\text {bnd }}$. The numerical evaluation of the respective domain integrals via integration point oversampling is outlined in Sec. 3.1.1.

The external load vector $\mathbf{f}$ in Eq. (12) consists of nodal point contributions of the type

$$
\mathbf{f}^{(I)}={\stackrel{\mathbf{A}}{n_{e}}}_{e=1} \int_{\Omega_{e}} \chi N^{(i)} \mathbf{b} \mathrm{dA}+{\stackrel{\mathbf{A}}{n_{e}}}_{e=1} \int_{\Gamma^{N}} N^{(i)} \mathbf{t} \mathrm{d} \mathbf{S} .
$$

Again, the respective domain integrals involving the characteristic function $\chi$ are discarded for elements within $\{\Omega\}^{\text {ext }}$, and allow for a standard evaluation considering elements within $\{\Omega\}^{\text {int }}$.

\footnotetext{
${ }^{4}$ The assembly operator $\mathbf{A}$ is a formal representation to account for the consideration and summation of local (elementwise) matrices at their appropriate locations within the corresponding global (system) matrices. For a detailed account on the formulation and implementation of finite element assembly routines we refer to [35].

${ }^{5}[\mathbf{I} \otimes \mathbf{I}]_{i j k l}=\delta_{i k} \delta_{j l},[\mathbf{I} \otimes \mathbf{I}]_{i j k l}=\delta_{i l} \delta_{j k}$.
} 


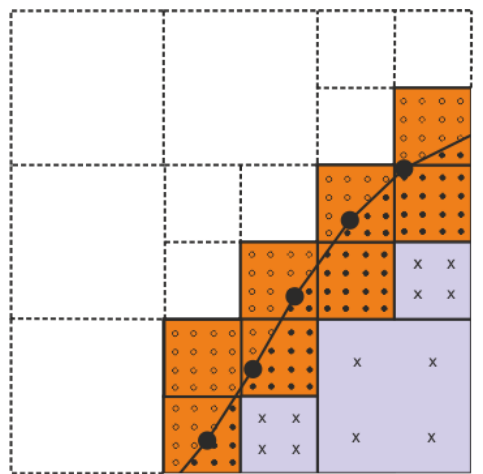

Figure 3: Evaluation of domain integrals via integration point oversampling: For all elements within $\left\{\Omega_{e}\right\}^{\text {bnd }}$ we supply $n_{\text {intp }}$ integration points, each of which is then checked to be interior or exterior to $\Gamma^{h}$. Typically, $n_{\text {intp }}$ is within the range $[25,100]$. All remaining domain integrals considering elements $\Omega_{e} \in\left\{\Omega_{e}\right\}^{\text {int }}$ are evaluated by means of a standard quadrature rule.

The numerical treatment of the domain integrals considering elements within $\left\{\Omega_{e}\right\}^{\text {bnd }}$ are taken into account via integration point oversampling as outlined in Sec. 3.1.1.

The remaining line integral contributions holding the natural (traction) boundary condition in Eq. (15) do only involve elements within $\{\Omega\}^{\text {bnd }}$ and are examined within Sec. 3.1.2.

\subsubsection{Domain integrals}

All domain integrals involving elements within $\{\Omega\}^{\text {bnd }}$ are characterized by a sharp intraelement discontinuity for the characteristic function $\chi$ across the physical domain boundary $\Gamma^{h}$. For the approximate numerical integration of the respective domain integrals in Eqs. (13) and (15), we rely on an oversupply of $n_{\text {int }}$ integration points considering all $\Omega_{e} \in\{\Omega\}^{\text {bnd }}$, cf. Fig. 3 . Each integration point is then checked to be interior or exterior to $\Gamma^{h}$ and the numerical integration is in the format

$$
\int_{\Omega_{e}} \chi(\boldsymbol{\xi}) f(\boldsymbol{\xi}) \mathrm{dA} \approx \sum_{k=1}^{n_{\text {intp }}} \chi_{i / e}\left(\boldsymbol{\xi}_{k}\right) f\left(\boldsymbol{\xi}_{k}\right) w_{k},
$$

where $\xi_{k}$ and $w_{k}$ denote the isoparametric position of the $k$-th integration point and its respective integration weight ${ }^{6}$. In Eq. (16), $\chi_{i / e}=1$ for all $\xi_{k}$ interior $\Gamma^{h}$ and $\chi_{i / e}=10^{-5}$ for all $\xi_{k}$ exterior $\Gamma^{h}$. The non-vanishing contribution from integration points exterior $\Gamma^{h}$ is a precaution to avoid the formation of a singular system stiffness matrix as a result of the assembly routine when only a small fraction of an intersected element lies within $\Gamma^{h}$. For the numerical examples within this contribution, we choose $n_{\text {intp }} \in[25,100]$.

\subsubsection{Natural boundary conditions}

For the numerical consideration of the natural (traction) boundary condition in Eq. (15), we need to evaluate the corresponding line integral along $\Gamma^{N}$ in a sequential manner involving all elements $\Omega_{e} \in\{\Omega\}^{\text {bnd }}$, cf. Figs. 4a-b.

\footnotetext{
${ }^{6}$ In Eq. (16), we assume the integration weight $w_{k}$ to contain the respective local Jacobian determinant to account for the transformation to the isoparametric reference domain already.
} 

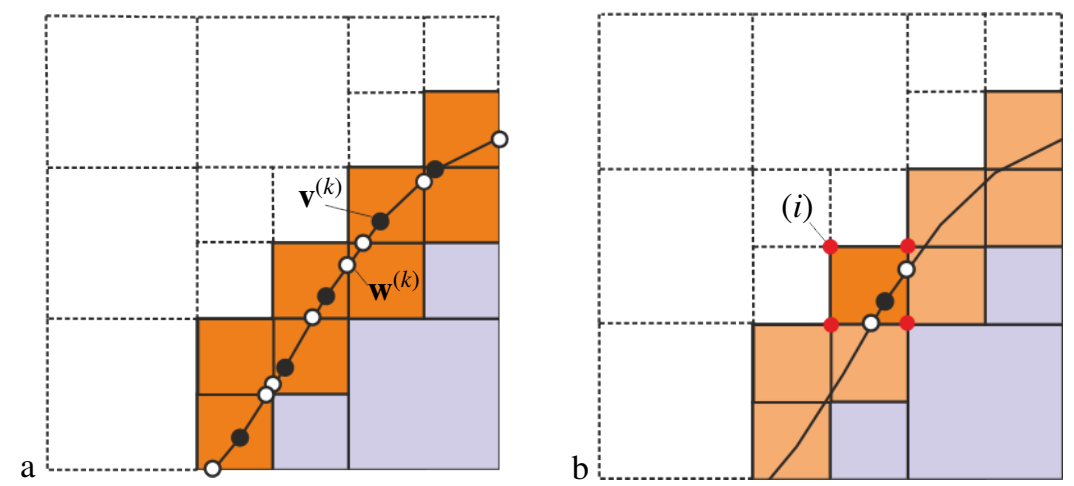

Figure 4: Sequential evaluation of line integrals considering natural and essential boundary conditions. a The individual line segments for elements $\Omega_{e} \in\{\Omega\}^{\text {bnd }}$ are specified by the boundary vertices $\mathbf{v}^{(k)}$ defining $\Gamma^{h}$ and the intersection vertices $\mathbf{w}^{(k)}$ that result from the intersection of $\Gamma^{h}$ and the element boundaries $\partial \Omega_{e} \in\{\Omega\}^{\text {bnd }}$. b The individual sub-integrals are then evaluated by use of a standard one-dimensional quadrature rule and assigning the respective contributions to the local nodal points $(i)$.

To this extent, we need not only consider the individual boundary segments $\Gamma_{s} \in \Gamma^{N}$ bound by its respective vertices $\mathbf{v}^{(k)}$, but also the intersection points $\mathbf{w}^{(k)}$ being specified by the intersection of $\Gamma^{N}$ and the individual element boundaries $\partial \Omega_{e} \in\left\{\Omega_{e}\right\}^{\text {bnd }}$. The line integral along $\Gamma^{N}$ is then obtained as a sum over all elements $\Omega_{e} \in\{\Omega\}^{\text {bnd }}$ that contribute at least one line segment $\left[\mathbf{v}^{(k)}, \mathbf{w}^{(k)}\right]$ to $\Gamma^{N}$

$$
\int_{\Gamma^{N}} N^{(i)} \mathbf{t} \mathrm{dS}=\sum_{k} \int_{\left\{\mathbf{v}^{(k)}\right\}}^{\left\{\mathbf{w}^{(k)}\right\}} N^{(i)} \mathbf{t} \mathrm{d} S,
$$

where a standard one-dimensional quadrature rule is used to evaluate the individual line (sub-) integrals $\left[\mathbf{v}^{(k)}, \mathbf{w}^{(k)}\right]$ and $i \in\left[1, n_{e n}\right]$.

\subsubsection{Essential boundary conditions}

In order to complete the Euler-Lagrange equations in (3), a set of essential boundary conditions $\overline{\mathbf{u}}$ for the unknown displacement function is specified on $\Gamma^{D}$. Since no degrees of freedom are assigned directly to $\Gamma^{h}$ within the embedded domain setting, we employ a penalty method to fulfil the essential boundary condition in a weak (or integral) sense. Specifically, we employ the penalty formulation outlined in [36]. Other methods to enforce essential boundary conditions in fictitious domain or mesh-free formulations include the use of Lagrange multipliers [31, 37], and the Nitsche method [38, 39].

For the penalty approach, the weak formulation of the boundary value problem in Eq. (6) is augmented by a penalty term considering the essential boundary condition on $\Gamma^{D}$

$$
W_{\chi}^{p}(\mathbf{u}, \delta \mathbf{u})=W_{\chi}(\mathbf{u}, \delta \mathbf{u})+\beta \int_{\Gamma^{D}}(\mathbf{u}-\overline{\mathbf{u}}) \delta \mathbf{u} \mathrm{d} \mathrm{S}=0,
$$

where $\beta$, typically in the range $\beta \in\left[10^{8}, 10^{10}\right]$, is the penalty parameter.

Upon discretization, we obtain a modified version of the matrix system of equations in (12) which is in the format

$$
[\mathbf{K}+\beta \mathbf{L}] \mathbf{u}^{h}=\mathbf{f}+\beta \mathbf{l}
$$


In Eq. (19) the penalty matrix $\mathbf{L}$ consists of dual nodal point contributions of the type

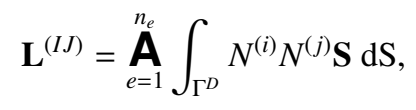

where the assembly operator spans over all elements within $\{\Omega\}^{\text {bnd }}$ that include segments $\Gamma_{s} \subset$ $\Gamma^{D}$. The component matrix $\mathbf{S}$ in (20), which enables to differentiate among all available degrees of freedom for a specific nodal point combination $(I J)$, is in the format

$$
\mathbf{S}=\left[\begin{array}{cc}
S_{x} & 0 \\
0 & S_{y}
\end{array}\right] .
$$

Correspondingly, $S_{x}=1$ or $S_{y}=1$ if the respective displacement component $u_{x}$ or $u_{y}$ is prescribed along $\Gamma^{D}$, and $S_{x / y}=0$ otherwise.

The penalty vector $\mathbf{l}$ in Eq. (19) consists of nodal point contributions of the type

$$
\mathbf{l}^{(I)}=\underset{e=1}{n_{e}} \int_{\Gamma^{D}} N^{(i)} \mathbf{S} \overline{\mathbf{u}}^{h} \mathrm{~d} \mathbf{S},
$$

where the vector $\overline{\mathbf{u}}^{h}=\left[\bar{u}_{x}, \bar{u}_{y}\right]^{T}$ holds the prescribed displacement components along $\Gamma^{D}$.

The line integrals in Eqs. (20) and (22) are evaluated in the same sequential manner as outlined for the natural boundary condition in Eq. (17) and by use of a standard one-dimensional quadrature rule.

\subsection{Reorientation scheme}

The staircase scheme outlined in Sec. 3.1 accounts for the geometric mismatch between the physical boundary $\Gamma^{h}$ and its embedding domain representation $\{\Omega\}^{\text {bnd }}$ by use of selective domain integration. For the reorientation scheme, we aim to relocate a set of nodal points in order to establish a layer of reoriented elements, the boundary of which then conforms to $\Gamma^{h}$.

In other words, we aim to overcome the presence of $\{\Omega\}^{\text {bnd }}$ by reorienting its elements $\Omega_{e}$ and assigning them either to $\{\Omega\}^{\text {int }}$ or $\{\Omega\}^{\text {ext }}$. Thereby, starting from the previously established regular mesh in Fig. 5a, the relocation procedure first entails the mapping of the respective closest nodal point $(I) \in\{\Omega\}^{\text {bnd }}$ onto each individual $\mathbf{v}^{(k)} \in \Gamma^{h}$. Subsequently, we consider an elementwise decisive closest point projection of remaining nodal points onto $\Gamma^{h}$. All elements affected by this relocation procedure are then assigned to $\left\{\Omega_{e}\right\}^{\text {int }}$ or $\left\{\Omega_{e}\right\}^{\text {ext }}$, based on their relative positioning against the physical domain boundary $\Gamma^{h}$.

However, in order to avoid poorly shaped or even inverted quadrilateral elements as a consequence of this relocation strategy, certain segments of $\Gamma^{h}$ may not be aligned with reoriented element boundaries, but rather intersect a quadrilateral diagonally, cf. Fig. 5b. In this case, the respective domain integral contributions of the triangular sub-domain interior $\Gamma^{h}$ are taken into account by use of a tailored quadrature rule for triangular domains, cf. Figs. 6a-b.

In order to compensate for the resulting disparity concerning mesh quality measures between (non-modified) regular-shaped elements interior $\Gamma^{h}$ and elements that are affected by the relocation procedure, we allow for a sequence of mesh smoothing operations. This involves the in-plane movement of previously mapped nodal points alongside $\Gamma^{h}$, as well as the relocation of nodal points in the proximity of $\Gamma^{h}$ towards their particular barycentre that is defined by adjoining element edges. For the present contribution, element quality is measured through a scaled Jacobian quantity $j \in[-1,1]$, where $j=1$ for regularly-shaped quadrilaterals irrespective of 
their geometric extent, and $j \leq 0$ for inverted quadrilaterals [40]. For the numerical examples in this contribution, we set the acceptable range for the element quality to $j \in[0.5,1]$.

Due to this nodal relocation strategy, a special treatment for the evaluation of the domain integrals within the assembly procedure of the stiffness matrix $\mathbf{K}$ is only necessary for elements that are intersected diagonally by the physical domain boundary $\Gamma^{h}$. All other quadrilaterals allow for an exact domain integration by use of a standard quadrature rule, or can be fully discarded in case of elements exterior $\Gamma^{h}$.

Considering quadrilaterals that are intersected diagonally by $\Gamma^{h}$, the domain integration allows for a geometric separation in the format

$$
\int_{\Omega_{e}} \chi(\xi) f(\xi) \mathrm{dA}=\int_{\Delta^{\mathrm{int}}\left\{\Omega_{e}\right\}} \chi(\boldsymbol{\xi}) f(\boldsymbol{\xi}) \mathrm{dA}+\int_{\Delta^{\operatorname{ext}\left\{\Omega_{e}\right\}}} \chi(\boldsymbol{\xi}) f(\boldsymbol{\xi}) \mathrm{dA}
$$

where $\Delta^{\text {int }}\left\{\Omega_{e}\right\}$ and $\Delta^{\text {ext }}\left\{\Omega_{e}\right\}$ denote the respective triangular sub-domain interior and exterior $\Gamma^{h}$. Therefore, the sharp intra-element discontinuity of the characteristic function $\chi$ across the physical domain boundary $\Gamma^{h}$ is taken into account through an equivalent subdivision of the respective element domain integral.

Now, since $\chi=0 \forall \mathbf{X} \in \Omega \backslash \mathcal{B}$, the domain integral contribution from $\Delta^{\mathrm{ext}}\left\{\Omega_{e}\right\}$ can be fully discarded, and we arrive at

$$
\int_{\Omega_{e}} \chi(\boldsymbol{\xi}) f(\boldsymbol{\xi}) \mathrm{dA}=\int_{\Delta^{\operatorname{int}}\left\{\Omega_{e}\right\}} f(\boldsymbol{\xi}) \mathrm{dA}=\sum_{k=1}^{n_{\text {intp }}} f\left(\hat{\boldsymbol{\xi}}_{k}\right) \hat{w}_{k}
$$

In Eq. (24), $\hat{\boldsymbol{\xi}}_{k}$ and $\hat{w}_{k}$ denote the isoparametric position of the $k$-th integration point and its respective integration weight considering an adequate quadrature rule for triangular (sub-)domains $[41,42]$. Similarly, considering the evaluation of line integrals for natural and essential boundary conditions, a non-standard treatment is only necessary for elements that are intersected diagonally by $\Gamma^{h}$. All other (interior) elements in the proximity of $\Gamma^{h}$ are aligned with the physical domain boundary and hence allow for a standard consideration of boundary conditions.

Natural boundary conditions for intersected elements are assembled as outlined for the staircase scheme in Sec. 3.1.2, except that the contributions to the load vector $\mathbf{f}$ stemming from the degrees of freedom at the respective exterior nodal point are equally assigned to the adjacent nodal points lying on $\Gamma^{h}$. Essential boundary conditions for intersected elements are taken into account by assigning the prescribed displacements along $\Gamma^{D}$ to the respective degrees of freedom at the exterior nodal point as well as the nodal points mapped onto $\Gamma^{h}$.

\section{Shape optimization}

Throughout this contribution, we aim to solve numerically a sequence of shape optimization problems in the general format

$$
\min _{\mathcal{B} \in \mathcal{U}^{a d}} \psi=\psi(\mathcal{B}, \mathbf{u}(\mathcal{B}))
$$

through a variation of the configuration $\mathcal{B}$ within the class of admissible shapes $\mathcal{U}^{a d}$. In the sequel, we consider $\mathcal{U}^{a d}$ to comprise all possible configurations of the evolving shape $\mathcal{B}$ within the bounds of the embedding domain $\Omega$.

Moreover, we assume the objective function $\psi$ to depend explicitly on the shape $\mathcal{B}$ as well as implicitly through the solution $\mathbf{u}$ of the state equation in (3). As a prerequisite for the efficient 

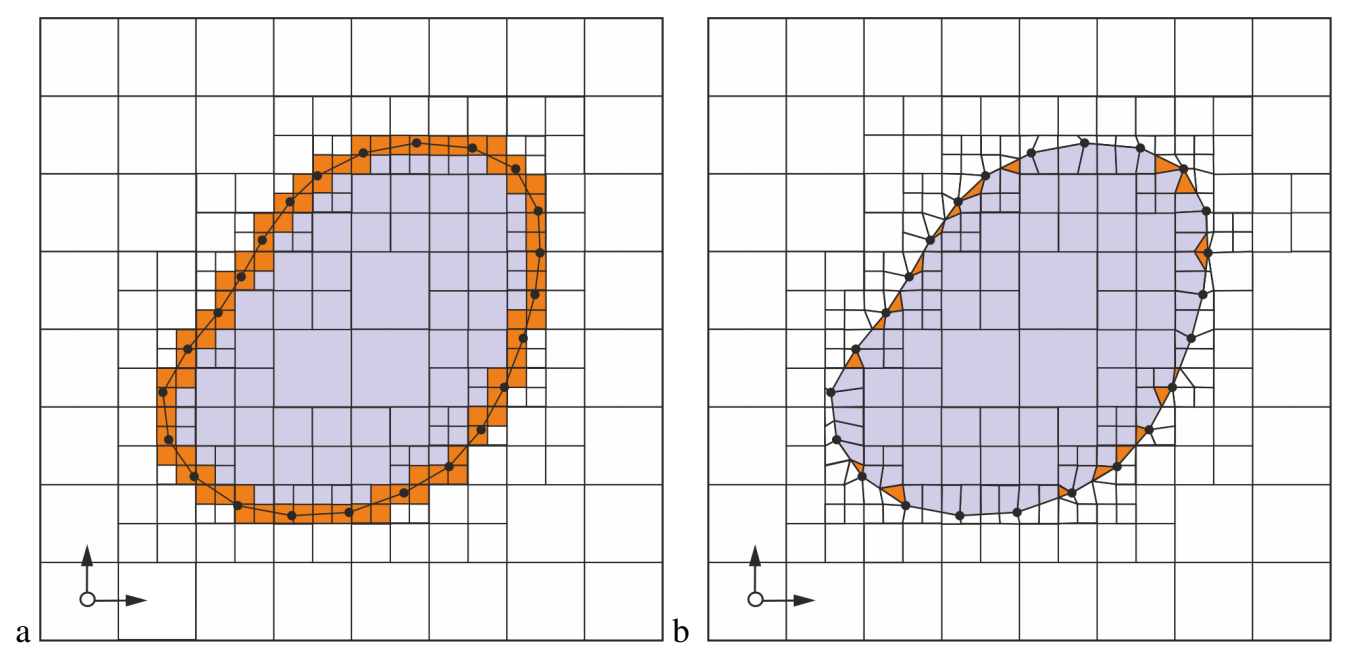

Figure 5: Outline of the nodal relocation strategy to obtain a layer of elements within $\Omega^{h}$ that is aligned with the physical domain boundary $\Gamma^{h}$. Starting from the adaptively refined regular mesh in $\mathbf{a}$, we obtain a locally reoriented mesh in $\mathbf{b}$ that includes elements aligned with $\Gamma^{h}$, and elements that are intersected diagonally by $\Gamma^{h}$ leading to triangular sub-domains on both sides of $\Gamma^{h}$.
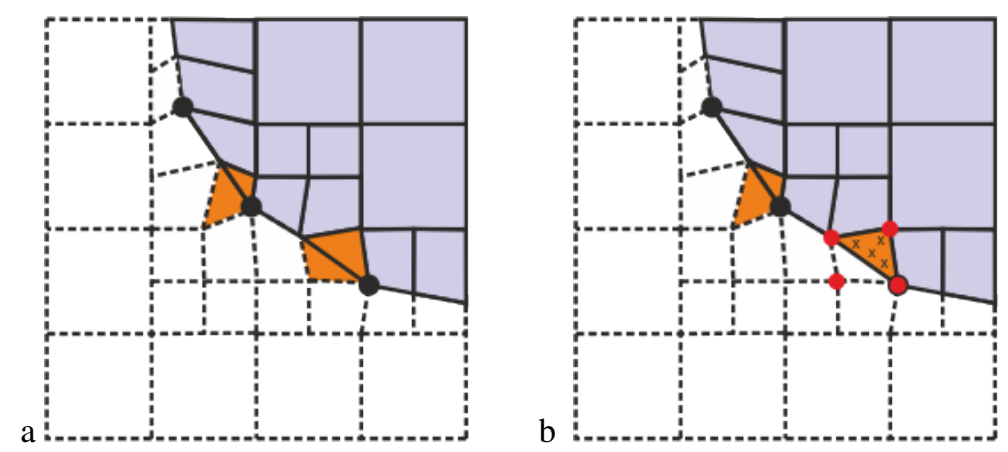

Figure 6: Evaluation of domain integrals within the reorientation scheme. In order to take into account the remaining geometric mismatch that is observed for quadrilateral elements that are intersected diagonally by $\Gamma^{h}$ in a, a selective domain integration is achieved employing a quadrature rule considering triangular (sub-)domains. b Thereby, the domain integration is restricted to the interior triangular sub-domain, whereas integral contributions from the exterior triangular sub-domain are discarded. 
numerical solution of (25), we need to derive the design sensitivity expression for the objective function $\psi$, which characterizes the change in $\psi$ for a design perturbation $\delta \mathbf{X}$ in $\mathcal{B}$ normal to $\Gamma$. In principle, the design sensitivity expression can either be derived for the continuous setting in Eq. (3) or for the discrete setting in Eq. (12), where we choose the former option since it allows for a more general derivation in the present context ${ }^{7}$. However, since the evaluation of the resulting design sensitivity expression is again realised within the discrete embedding domain setting outlined in Sec. 3, this approach is not free from mesh dependency effects.

Technically, domain variations of $\mathcal{B}$ are realised through a direct modification of vertex coordinates $\mathbf{v}^{(k)} \in \Gamma^{h}$, and not altering the boundary connectivity that is given in terms of $\Gamma_{s} \subset \Gamma^{h}$. For every vertex $\mathbf{v}^{(k)}$ the local outward unit normal $\mathbf{n}^{(k)}$ is obtained by averaging the outward normal vectors of its adjacent boundary segments $\Gamma_{s}$.

In a way similar to the subdivision of the boundary $\Gamma$ into $\Gamma^{D}$ and $\Gamma^{N}$ when specifying the state equation in (3), we consider $\Gamma^{h}$ to be subdivided in the course of the optimization. Thereby, we denote by $\Gamma^{V}$ the boundary portion that is to be varied during the course of optimization and by $\Gamma^{P}$ the prescribed or non-variable portion of $\Gamma^{h}$. However, if not stated otherwise, we still allow for the in-plane (or tangential) movement of vertices $\mathbf{v}^{(k)}$ alongside $\Gamma^{P}$. In fact, this mechanism is a technical requirement to avoid the premature termination of the optimization procedure due to a self-intersection of $\Gamma^{h}$.

\subsection{Design sensitivity analysis}

As a mathematical framework to account for general shape variations of the continuum body $\mathcal{B}$, we consider a family of one-to-one domain perturbations $T$ that maps continuum points from their reference position $\mathbf{X}$ onto their current placement $\mathbf{x}_{\tau}$

$$
T: \mathbf{X} \mapsto \mathbf{x}_{\tau}(\mathbf{X})
$$

where $\tau$ is a time-like parameter $\tau \in[0, t]$ and we denote by $\mathcal{B}_{\tau}$ and $\Gamma_{\tau}$ the perturbed domain and its boundary, respectively

$$
\mathcal{B}_{\tau}=T(\mathcal{B}, \tau), \quad \Gamma_{\tau}=T(\Gamma, \tau) .
$$

Using a linear Taylor's expansion at $\tau=0$, the placement $\mathbf{x}_{\tau}$ is in the format

$$
\mathbf{x}_{\tau}=T(\mathbf{X}, \tau)=\mathbf{X}+\tau \boldsymbol{\theta}(\mathbf{X}),
$$

where $\boldsymbol{\theta}$ denotes the design velocity field $[43,44]$. The formal representation in (28), the socalled Hadamard boundary variation method [45], allows for the evaluation of the material (or total) derivative $\{\dot{\bullet}\}=\mathrm{D}\{\bullet\} / \mathrm{D} \tau$ of scalar and vector-valued field variables $a=a\left(\mathbf{x}_{\tau}, \tau\right)$ and $\mathbf{a}=\mathbf{a}\left(\mathbf{x}_{\tau}, \tau\right)$ in the direction of $\boldsymbol{\theta}$

$$
\begin{gathered}
\dot{a}=a^{\prime}+\nabla a \cdot \boldsymbol{\theta} \\
\dot{\mathbf{a}}=\mathbf{a}^{\prime}+[\nabla \mathbf{a}] \boldsymbol{\theta} .
\end{gathered}
$$

The material derivative formulae in Eq. (29) both consist from a spatial derivative $\{\bullet\}^{\prime}=\partial\{\bullet\} / \partial \tau$ and a convective derivative $\nabla\{\bullet\} \cdot \theta$ in terms of the design velocity field $\boldsymbol{\theta}$.

\footnotetext{
${ }^{7}$ Strictly speaking, the result of the continuous sensitivity analysis is general in the sense that it is not tailored to the specifics of a certain numerical analysis scheme. The resulting shape sensitivity expression may be used for optimization algorithms that rely on conforming finite element methods, embedding domain techniques, or boundary element methods.
} 
At this point, let us consider an objective functional $\psi$ that comprises from volume and surface integral contributions of the general type

$$
\psi_{\tau}=\int_{\mathcal{B}_{\tau}} G \mathrm{dA}+\int_{\Gamma_{\tau}} g \mathrm{dS}
$$

where $G=G(\mathbf{u}), g=g(\mathbf{u}, \mathbf{t})$ on $\Gamma^{N}$, and $g=g\left(\mathbf{u}, \mathbf{t}_{0}\right)$ on $\Gamma^{D}$ with reaction forces $\mathbf{t}_{0}$.

The material derivative of $\psi$ at $\tau=0$ is obtained through use of the Reynolds' transport theorem and using the material derivative formula (29) for the integrands $G$ and $g$

$$
\begin{aligned}
\dot{\psi} & =\int_{\mathcal{B}}[\dot{G}+G \nabla \cdot \boldsymbol{\theta}] \mathrm{dA}+\int_{\Gamma}[\dot{g}+g H[\boldsymbol{\theta} \cdot \mathbf{n}]] \mathrm{dS} \\
& =\int_{\mathcal{B}} G^{\prime} \mathrm{dA}+\int_{\Gamma} G[\boldsymbol{\theta} \cdot \mathbf{n}] \mathrm{d} \mathrm{S}+\int_{\Gamma} g^{\prime}+[\nabla g \cdot \mathbf{n}+g H][\boldsymbol{\theta} \cdot \mathbf{n}] \mathrm{dS},
\end{aligned}
$$

where $H=\nabla \cdot \mathbf{n}$ denotes the curvature of $\Gamma$ in $\mathcal{R}^{2}$. Since $G^{\prime}=G_{, \mathbf{u}} \mathbf{u}^{\prime}$ and equally $g^{\prime}=g_{, \mathbf{u}} \mathbf{u}^{\prime}+g_{, \mathbf{t}} \mathbf{t}^{\prime}$, it remains to determine the spatial derivatives $\mathbf{u}^{\prime}$ and $\mathbf{t}^{\prime}$, or their respective material derivatives since both are interrelated via Eq. (29).

To this extent, by use of the adjoint variable method for design sensitivity analysis $[46,47,48]$, we first introduce a weak form $W=W\left(\mathbf{u}, \mathbf{u}^{a}\right)$ of the boundary value problem (3), in which the adjoint state field $\mathbf{u}^{a}$ is employed as a test function for the state equation

$$
W\left(\mathbf{u}, \mathbf{u}^{a}\right)=-\int_{\mathcal{B}} \boldsymbol{\sigma}: \boldsymbol{\epsilon}^{a} \mathrm{dA}+\int_{\mathcal{B}} \mathbf{b} \cdot \mathbf{u}^{a} \mathrm{dA}+\int_{\Gamma^{N}} \mathbf{t} \cdot \mathbf{u}^{a} \mathrm{dS}=0,
$$

and $\boldsymbol{\epsilon}^{a}=\nabla^{\mathrm{sym}} \mathbf{u}^{a}$.

Subsequently, the weak form of the state equation in Eq. (32) is added to the objective functional $\psi$ in (30) to define an augmented objective functional $L$ in the format

$$
L=\psi+W=\int_{\mathcal{B}} \bar{G} \mathrm{dA}+\int_{\Gamma^{N}} \bar{g} \mathrm{dS}+\int_{\Gamma^{D}} \bar{g} \mathrm{dS},
$$

where the respective integrands amount to

$$
\begin{array}{ll}
\bar{G}=G-\boldsymbol{\sigma}: \boldsymbol{\epsilon}^{a}+\mathbf{b} \cdot \mathbf{u}^{a} & \text { in } \mathcal{B} \\
\bar{g}=g+\mathbf{t} \cdot \mathbf{u}^{a} & \text { on } \Gamma^{N} \\
\bar{g}=g & \text { on } \Gamma^{D} .
\end{array}
$$

In fact, $W=W\left(\mathbf{u}, \mathbf{u}^{a}\right)$ in Eq. (33) is employed as an additional constraint to the optimization problem in (25), where the corresponding Lagrange multiplier $\mathbf{u}^{a}$ is determined by the solution of an adjoint boundary value problem on $\mathcal{B}$.

The setting of this adjoint boundary value problem is a direct result from the requirement that the (physical) state equation (3) is not only to be fulfilled for the current domain $\mathcal{B}$, but also for the perturbed domain $\mathcal{B}_{\tau}$. Hence, the augmented objective functional $L$ in (33) is required to be stationary with respect to variations of the primary state field $\mathbf{u}$ and the adjoint state field $\mathbf{u}^{a}$. This requirement is formally taken into account by considering the time derivative $\dot{L}$ of the augmented objective functional, and setting the collection of all terms involving implicit derivatives $\mathbf{u}^{\prime}$ and 
$\mathbf{u}^{a \prime}$ equal to zero. Accordingly, by use of Eqs. (33), (34) and (31), we obtain

$$
\begin{aligned}
\dot{L}= & \int_{\Gamma} \bar{G}[\boldsymbol{\theta} \cdot \mathbf{n}] \mathrm{d} \mathrm{S}+\int_{\Gamma}[\nabla \bar{g} \cdot \mathbf{n}+\bar{g} H][\boldsymbol{\theta} \cdot \mathbf{n}] \mathrm{d} \mathrm{S}+ \\
& \int_{\mathcal{B}} \mathbf{b}^{\prime} \cdot \mathbf{u}^{a} \mathrm{dA}+\int_{\Gamma^{N}}\left[g_{, \mathbf{t}}+\mathbf{u}^{a}\right] \cdot \mathbf{t}^{\prime} \mathrm{d} \mathrm{S}+\int_{\Gamma^{D}} g_{, \mathbf{u}} \cdot \overline{\mathbf{u}}^{\prime}-\overline{\mathbf{u}}^{a} \cdot \mathbf{t}_{0}^{\prime} \mathrm{d} \mathbf{S}+ \\
& Q\left(\mathbf{u}^{\prime}, \mathbf{u}^{a \prime}\right),
\end{aligned}
$$

where the prescribed adjoint solution variable $\overline{\mathbf{u}}^{a}=-g_{\mathbf{t}_{0}}$ on $\Gamma^{D}$ and $Q$ in (35) contains all terms involving implicit derivatives considering the primary and adjoint state fields

$$
\begin{aligned}
Q\left(\mathbf{u}^{\prime}, \mathbf{u}^{a \prime}\right)=0= & \left(-\int_{\mathcal{B}} \boldsymbol{\sigma}: \boldsymbol{\epsilon}^{a \prime} \mathrm{dA}+\int_{\mathcal{B}} \mathbf{b} \cdot \mathbf{u}^{a \prime} \mathrm{dA}+\int_{\Gamma^{N}} \mathbf{t} \cdot \mathbf{u}^{a \prime} \mathrm{dS}\right)+ \\
& \left(-\int_{\mathcal{B}} \boldsymbol{\epsilon}^{a}: \boldsymbol{\sigma}^{\prime} \mathrm{dA}+\int_{\mathcal{B}} \mathbf{G}_{, \mathbf{u}} \cdot \mathbf{u}^{\prime} \mathrm{dA}+\int_{\Gamma^{N}} g_{, \mathbf{u}} \cdot \mathbf{u}^{\prime}\right) .
\end{aligned}
$$

By inspection, we find that the expression within the first pair of brackets in Eq. (36) vanishes identically since it represents a weak form of the primary state equation (3) in which $\mathbf{u}^{a \prime}$ is employed as a test or virtual displacement function. By use of the stress-strain relation $\sigma^{\prime}=\mathbb{E}$ : $\boldsymbol{\epsilon}^{\prime}$, where $\boldsymbol{\epsilon}^{\prime}=\nabla^{\mathrm{sym}} \mathbf{u}^{\prime}$, and considering the major symmetry of the elasticity tensor $\mathbb{E}$, we obtain

$$
\epsilon^{a}: \sigma^{\prime}=\epsilon^{\prime}: \sigma^{a}
$$

Then, by use of Eq. (37), and introducing distributed body forces $\mathbf{b}^{a}$ as well as surface tractions $\mathbf{t}^{a}$ for the adjoint structure via

$$
\mathbf{b}^{a}=G_{, \mathbf{u}} \text { in } \mathcal{B}, \quad \mathbf{t}^{a}=g_{, \mathbf{u}} \text { on } \Gamma^{N},
$$

we obtain a weak form $W^{\text {adj }}$ of the adjoint boundary value problem with solution $\mathbf{u}^{a}$

$$
W^{\mathrm{adj}}=W^{\mathrm{adj}}\left(\mathbf{u}^{a}, \mathbf{u}^{\prime}\right)=-\int_{\mathcal{B}} \boldsymbol{\sigma}^{a}: \boldsymbol{\epsilon}^{\prime} \mathrm{dA}+\int_{\mathcal{B}} \mathbf{b}^{a} \cdot \mathbf{u}^{\prime} \mathrm{dA}+\int_{\Gamma^{N}} \mathbf{t}^{a} \cdot \mathbf{u}^{\prime} \mathrm{d} \mathrm{S}=0 .
$$

Thereby, Eq. (35) reduces to the sought expression for the total derivative of the objective functional $\psi$ in the direction of $\boldsymbol{\theta}$.

For clarification, let us consider a standard compliance functional $C$ in the format

$$
C=\int_{\mathcal{B}} \mathbf{b} \cdot \mathbf{u} \mathrm{dA}+\int_{\Gamma^{N}} \mathbf{t} \cdot \mathbf{u} \mathrm{d} \mathrm{S}
$$

such that $G=\mathbf{b} \cdot \mathbf{u}$ in $\mathcal{B}, g=\mathbf{t} \cdot \mathbf{u}$ on $\Gamma^{N}$, and $g=0$ on $\Gamma^{D}$ in Eq. (30). For the adjoint loads we obtain

$$
\mathbf{b}^{a}=G_{, \mathbf{u}}=\mathbf{b}, \quad \mathbf{t}^{a}=g_{, \mathbf{u}}=\mathbf{t},
$$

and hence the problem is self-adjoint in the sense that the adjoint solution variable equals the primary (or physical) solution variable: $\mathbf{u}^{a}=\mathbf{u}$. If we further assume the surface traction $\mathbf{t}$ and 
the distributed body force $\mathbf{b}$ not to vary with pseudo-time $\tau$, we arrive at the total derivative for the compliance functional in the direction of $\boldsymbol{\theta}$

$$
\dot{C}=\int_{\Gamma^{N}}[2 \mathbf{b} \cdot \mathbf{u}+2 H[\mathbf{t} \cdot \mathbf{u}]+2 \mathbf{n} \cdot \nabla[\mathbf{t} \cdot \mathbf{u}]-\boldsymbol{\sigma}: \boldsymbol{\epsilon}][\boldsymbol{\theta} \cdot \mathbf{n}] \mathrm{d} S+\int_{\Gamma^{D}} \boldsymbol{\sigma}: \boldsymbol{\epsilon}[\boldsymbol{\theta} \cdot \boldsymbol{n}] \mathrm{dS} .
$$

Similarly, if we consider a volume-type integral for the current shape $\mathcal{B}$

$$
V=\int_{\mathcal{B}} 1 \mathrm{dA}
$$

its total derivative in the direction of $\boldsymbol{\theta}$ is expressed through

$$
\dot{V}=\int_{\Gamma} 1[\boldsymbol{\theta} \cdot \mathbf{n}] \mathrm{dS} .
$$

\subsection{Descent directions and geometric regularization}

Generally speaking, the total derivative of an objective functional $\psi$ considering a domain variation in the direction of $\boldsymbol{\theta}$ is in the format

$$
\dot{\psi}=\int_{\Gamma} v[\boldsymbol{\theta} \cdot \mathbf{n}] \mathrm{dS},
$$

where the scalar integrand $v$ depends on the objective under consideration, cf. Eqs. (42) and (44). Moreover, the total derivative in Eq. (45) relies only on the normal component of the design velocity field: $[\boldsymbol{\theta} \cdot \mathbf{n}]$. Indeed, this notion goes back to a classical result in shape optimization theory, cf. also the detailed account in [19] and references therein, or the classic treatment [49]. The most direct approach to obtain a suitable descent direction $\boldsymbol{\theta}$ for the use in numerical algorithms of shape optimization is to employ the outward unit normal to $\Gamma$ times the (negative) scalar integrand $v$

$$
\mathbf{d}=-v \mathbf{n} \text {. }
$$

Then, establishing the design velocity field via $\boldsymbol{\theta}=\mathbf{d}$ and considering a small $(\tau \rightarrow 0)$ neighbourhood of $\mathcal{B}$, we obtain the relation

$$
\psi\left(\mathcal{B}_{\tau}\right)=\psi(\mathcal{B})-\tau \int_{\Gamma} v^{2} \mathrm{dS}+O\left(\tau^{2}\right)
$$

where $O\left(\tau^{2}\right)$ contains higher-order terms of $\tau$ and $\lim _{\tau \rightarrow 0} O\left(\tau^{2}\right) / \tau=0$. Thereby, we conclude that $\psi\left(\mathcal{B}_{\tau}\right)<\psi(\mathcal{B})$ and $\mathbf{d}$ in Eq. (46) denotes the steepest descent direction.

However, due to the polygonal representation of the domain boundary $\Gamma^{h}$, a direct use of the steepest descent direction for domain variations may easily result in irregular or even selfintersecting boundaries. This is also true on the condition that the initial domain boundary fulfils certain smoothness assumptions. One such example is given for a shape $\mathcal{B}$ that features a rightangled corner and is subject to a uniform volume shrinkage alongside its boundary $\Gamma^{h}$. After several domain updates of the type (28) and depending on the choice of the step-length parameter $\tau$, we observe an intersection among the two boundary segments $\Gamma_{s}$ adjacent to the corner node $\mathbf{v}^{(k)}$, cf. Fig. 7.

There exist several approaches in the literature that aim to avoid such geometric distortion phenomena $[50,51,52,53]$, where the common objective is to obtain from the steepest descent 
direction $\mathbf{d}$ in (46) a more regular descent direction $\widehat{\mathbf{d}}$, which is then used to alter the domain boundary via $\boldsymbol{\theta}=\widehat{\mathbf{d}}$ within a numerical algorithm.

For the present contribution, we resort to a geometrical regularization technique common to applications in computer graphics, in particular the field of digital image recognition and object detection, namely the Laplacian averaging technique $[54,55]$. In this method, geometric regularity is improved by moving every vertex $\mathbf{v}^{(k)}$ towards the central position $\tilde{\mathbf{v}}^{(k)}$ that is defined by averaging the position vectors of its adjacent vertices $\mathbf{v}^{(k-1)}$ and $\mathbf{v}^{(k+1)}$, cf. Fig. 8 .

The nodal regularization vector $\mathbf{r}^{(k)}$ is then obtained as the difference between $\tilde{\mathbf{v}}^{(k)}$ and $\mathbf{v}^{(k)}$

$$
\mathbf{r}^{(k)}=\tilde{\mathbf{v}}^{(k)}-\mathbf{v}^{(k)}
$$

and we denote by $\mathbf{r}$ the respective global vector that contains all individual contributions $\mathbf{r}^{(k)}$

$$
\mathbf{r}=\left[\mathbf{r}^{(0)}, \mathbf{r}^{(1)}, \ldots, \mathbf{r}^{\left(n_{v}-1\right)}\right]^{T} .
$$

Along the same line, we denote by $\mathbf{d}^{(k)}$ the nodal steepest descent direction that is obtained from the evaluation of $v$ and the outward unit normal $\mathbf{n}^{(k)}$

$$
\mathbf{d}^{(k)}=-v \mathbf{n}^{(k)} .
$$

Correspondingly, we denote by $\mathbf{d}$ the global vector that contains all individual contributions $\mathbf{d}^{(k)}$

$$
\mathbf{d}=\left[\mathbf{d}^{(0)}, \mathbf{d}^{(1)}, \ldots, \mathbf{d}^{\left(n_{v}-1\right)}\right]^{T} .
$$

Now, in order to obtain a more regular descent direction, the main idea is to consider a superposition of the regularization vector $\mathbf{r}$ and the steepest descent direction $\mathbf{d}$ in the format

$$
\widehat{\mathbf{d}}=(1-\alpha) \mathbf{d}+\alpha \mathbf{r},
$$

where $\alpha \in(0,1)$ is a scalar parameter to weigh the individual contributions. Still, to observe a descent in the objective functional $\psi$ when employing $\widehat{\mathbf{d}}$ as the design velocity field, we require

$$
\cos \angle(\mathbf{d}, \widehat{\mathbf{d}})=\frac{\mathbf{d} \cdot \widehat{\mathbf{d}}}{|\mathbf{d}||\widehat{\mathbf{d}}|} \geq \eta,
$$

where $\eta \in(0,1)$, cf. the schematic representation in 2-parametric design space in Fig. 9.

From a technical perspective, we employ an adaptive procedure for the choice of the weighting parameter $\alpha$ and the angular threshold value $\eta$ throughout the course of optimization. Thereby, we specify up-front the required sufficient decrease condition in terms of $\eta$ in Eq. (53). Then, for every design iteration, we start from a weighting factor $\alpha=1$ and reduce it by fractions of $\Delta \alpha=0.05$ until the sufficient decrease condition in (53) is fulfilled by $\widehat{\mathbf{d}}$. In doing so, we aim to establish the most regular descent direction that still fulfils the sufficient decrease condition in terms of $\eta$. In addition, we reserve the mechanism of increasing $\eta$ to sharpen the sufficient decrease condition at later design iterations. This is primary a tool to enhance the speed of convergence in the case that the current shape design $\mathcal{B}$ lies in the vicinity of the sought-after optimal shape design.

After all, in order to allow for (induced) in-plane movements of vertices $\mathbf{v}^{(k)}$ alongside $\Gamma^{h}$, we propagate the design velocity field $\widehat{\mathbf{d}}$ by solving an additional linear system of equations

$$
\mathbf{H} \boldsymbol{\theta}=\widehat{\mathbf{d}} \text {. }
$$





Figure 7: An arbitrary shape design $\mathcal{B}$ featuring a right-angled corner at $\mathbf{v}^{(k)}$ is subject to a uniform volume shrinkage through $\boldsymbol{\theta}=-\mathbf{n}$. Depending on the choice of the step-length parameter $\tau$, a domain update procedure yields an intersection between adjacent boundary segments $\Gamma_{s} \subset \Gamma^{h}$ after several iterations.
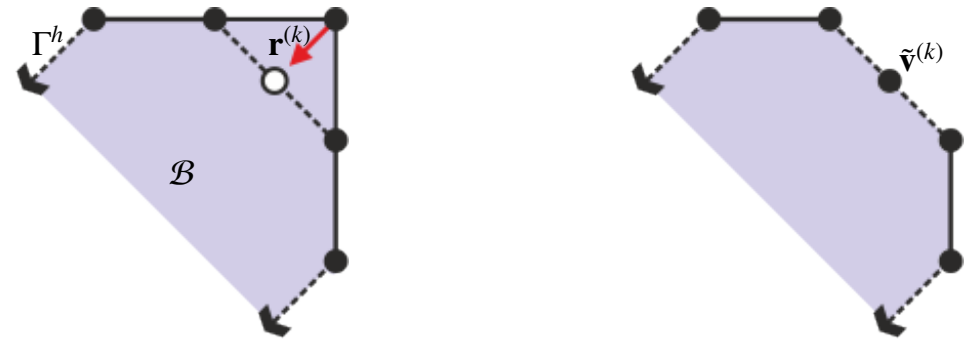

Figure 8: Local geometric regularization by use of Laplacian averaging. Vertices $\mathbf{v}^{(k)} \in \Gamma^{h}$ are shifted towards the central position $\tilde{\mathbf{v}}^{(k)}$ that is obtained by averaging the position vectors of their respective adjacent vertices $\mathbf{v}^{(k-1)}$ and $\mathbf{v}^{(k+1)}$.

The coefficient matrix $\mathbf{H}$ of dimension $2 n_{v} \times 2 n_{v}$ in (54) consists of dual vertex combinations of the type

$$
\mathbf{H}^{(K L)}=\boldsymbol{A}_{s=1}^{n_{s}} \int_{\Gamma_{s}} \mathbf{h}^{(k l)} \mathrm{dS},
$$

where the individual contributions $\mathbf{h}^{(k l)}$ from each segment $\Gamma_{s} \in \Gamma^{h}$ are in the format

$$
\mathbf{h}^{(k l)}=\left[\begin{array}{cccc}
1 & 0 & -1 & 0 \\
0 & 1 & 0 & -1 \\
-1 & 0 & 1 & 0 \\
0 & -1 & 0 & 1
\end{array}\right] .
$$

For the solution of the linear system of equations in (54), both the coefficient matrix $\mathbf{H}$ and the right-hand side vector $\widehat{\mathbf{d}}$ are subject to per-vertex constraints that are imposed from the definition of the non-variable boundary portion $\Gamma^{P}$. Then, by construction, the design velocity field $\boldsymbol{\theta}$ does not solely constitute from the non-zero entries of $\widehat{\mathbf{d}}$, but also from induced movements of vertices $\mathbf{v}^{(k)}$ due to the solution of (54), cf. the schematic one-dimensional representation given in Fig. 10.

\subsection{Algorithmic design for constrained optimization}

For the numerical examples in this contribution, we consider general problems of shape optimization as outlined in (25), and subject to one inequality constraint $g=g(\mathcal{B}, \mathbf{u}(\mathcal{B}))$

$$
\min _{\mathcal{B} \in \mathcal{U}^{a d}} \psi, \quad \text { s.t. } g \leq \bar{g}
$$




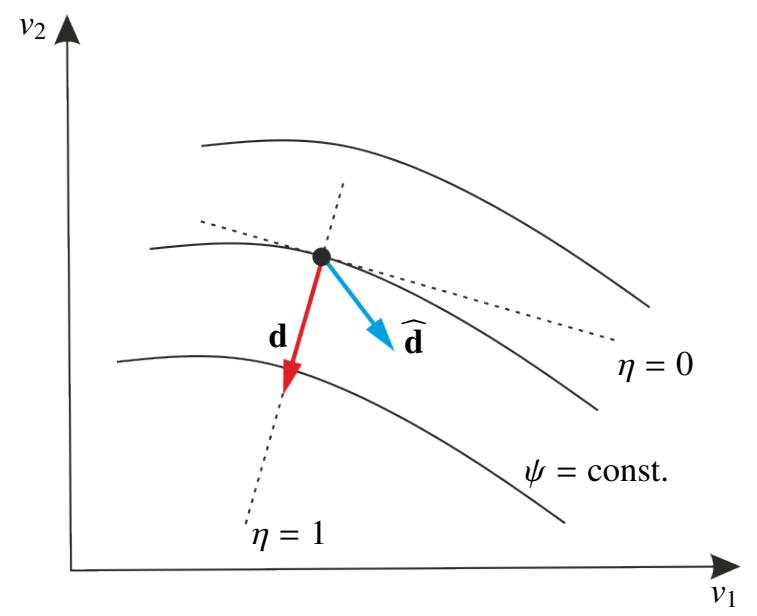

Figure 9: Schematic representation of the sufficient decrease condition (53) in 2-parametric design space. The steepest descent direction $\mathbf{d}$ is oriented normal to the objective isoline $\psi=$ const., and we require the combined descent direction $\widehat{\mathbf{d}}$ to inclose a maximum angle with the steepest descent in terms of a user-controlled variable $\eta$.

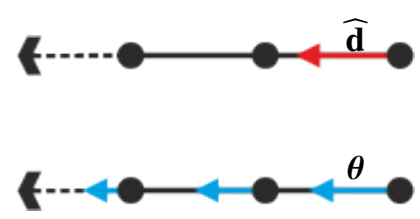

Figure 10: One-dimensional representation concerning the distribution of the combined descent direction $\widehat{\mathbf{d}}$ among adjoining vertices $\mathbf{v}^{(k)}$ through solution of the linear system of equations in (54). The resulting design velocity field $\boldsymbol{\theta}$ comprises induced movements of vertices $\mathbf{v}^{(k)}$ that circumvent the self-intersection or geometric inversion of neighbouring boundary segments $\Gamma_{s}$. 
where $\bar{g}$ is a user-specified upper limit for the constraint function $g$. In principle, there exist two classes of numerical algorithms for the solution of the constrained optimization problem in (57). On the one hand, indirect methods, such as penalty and Lagrange multiplier formulations, combine the objective and constraint function to form one single augmented function [56, 57]. This augmented function is then subject to a minimization procedure by use of basic descent algorithms considering unconstrained optimization problems [58]. One can show that the solution of this unconstrained optimization problem is also a solution of (57), and hence the constraint $g$ is fulfilled for the optimal design arrived at, even though it may be violated during the course of optimization.

On the contrary, direct methods, such as interior-point or barrier-type algorithms, aim to fulfil the constraint function at all times during the course of the optimization [59,60]. This is achieved by establishing descent directions that do not only lead to a reduction in the objective, but are also feasible in the sense that they do not violate the constraint.

For the present contribution, we resort to a numerical algorithm within the class of indirect methods, namely the augmented Lagrangian formulation by Rockafellar [61, 62]. However, when establishing the descent direction $\widehat{\mathbf{d}}$ from the combination of the steepest descent $\mathbf{d}$ and the regularization vector $\mathbf{r}$ in (52), we effectively adopt one key principle of direct methods, which is the attempt to project the respective descent direction into the feasible design space region. Thereby, the regularization technique in use may be regarded as a hidden constraint to the optimization problem in (57), which however is not strictly (or explicitly) enforced.

The main consideration of the augmented Lagrangian formulation at hand is to transform the constrained optimization problem (57) into a sequence $k=1, \ldots, n$ of unconstrained minimization problems introducing the augmented objective $M$ in the format

$$
M^{k}=M^{k}(\mathcal{B}, \mathbf{u}, \varphi, c)=\psi+[4 c]^{-1}\left[\vartheta^{2}-\varphi\right],
$$

where $c$ and $\varphi$ denote the penalty parameter and the Lagrange multiplier estimate for the $k$-th minimization sub-problem, respectively. The newly introduced function $\vartheta$ in (58) is used to assess the constraint condition for the current shape design

$$
\vartheta=\max \{\varphi-2 c \hat{g}, 0\}
$$

where $\hat{g}=g-\bar{g}$. At this point, it remains to outline a solution procedure for the unconstrained optimization problem considering $M^{k}$ in (58), as well as to specify the continuation of the solution procedure for the constrained optimization problem via $M^{k+1}$. The latter is achieved by an update of the Lagrange multiplier estimate $\varphi$, and an enforcement of the penalty parameter $c$ for the definition of $M^{k+1}$ via

$$
\varphi \leftarrow \vartheta, \quad c \leftarrow 2 c .
$$

Considering the solution of the $k$-th unconstrained minimization problem in terms of $M^{k}$, we employ a descent algorithm on the basis of the derivations in Sec. 4.2. Thereby, we first evaluate separately the shape derivative expressions for the objective $\psi$ and the constraint function $g$ that are involved in the formulation of $M^{k}$ in (58). As a result, we can provide the steepest descent direction $\mathbf{d}$ for the augmented objective, and in a subsequent step, we obtain $\widehat{\mathbf{d}}$ through the combination of $\mathbf{d}$ and the regularization vector $\mathbf{r}$ in (52).

By solution of the linear system of equations in (54), we establish a feasible design velocity field $\boldsymbol{\theta}$ for the shape variation of $\Gamma^{h}$ via (28). A basic backtracking line search algorithm is used to determine the first possible step-length parameter $\bar{\tau}$ out of the sequence $\left\{1, \tau, \tau^{2}, \tau^{3}, \ldots\right\}$ that fulfils 
a minimum decrease requirement [57] relating $M^{k}(\mathcal{B})$ and $M^{k}\left(\mathcal{B}_{\tau}\right)$, where $\tau \in(0,1)$.

Only then, we perform the actual domain update and preset the newly established shape design $\mathcal{B}_{\tau}$ as a starting point for the next iteration: $\mathcal{B}^{(i+1)} \leftarrow \mathcal{B}_{\tau}^{(i)}$. The above design update procedure for the solution of the unconstrained optimization problem is repeated until we meet a termination criterion in which we assess the relative function decrease in $M^{k}$, or we exceed a maximum number of design iterations $i$.

\section{Numerical examples}

At this point, let us examine the capabilities of the optimization algorithm outlined in Sec. 4 by means of three numerical studies. Specifically, we aim to investigate the intrinsic quality of the optimal shapes arrived at. This is to consider the effect of the regularization vector $\mathbf{r}$ on the shape perturbation, and the choice of the respective embedding domain discretization technique for the solution of the physical state problem.

The parametrization $\Gamma^{h}$ of the initial guess for each optimal design problem is obtained by positioning vertices $\mathbf{v}^{(k)}$ equidistantly alongside the domain boundary $\Gamma$. All numerical examples in this work are implemented using the deal.ii finite element library [63, 64, 65]. All computations rely on basic isoparametric linear finite element shape functions. As a consequence of the tracking procedure in Eq. (11) hanging node (or multi-point) constraints are to be considered between neighbouring finite elements showing different levels of refinement.

\subsection{L-shape under uniaxial loading}

To begin with, we consider the L-shape domain depicted in Fig. 11a. The domain is constrained in the $x$-direction at the left-hand edge, and is equipped with symmetry boundary conditions in the $y$-direction on the bottom edge. As a loading, we consider a uniform tension in the positive $x$-direction that is applied on the bottom right-hand edge. The objective of this study is to minimize the volume $\mathcal{V}$ of the domain while keeping the compliance $C$ constant. From an engineering point of view, the trivial solution to this optimal design problem is a bar of uniform cross-section.

For the solution of the physical state problem throughout the course of the optimization, we employ the reorientation scheme. In order not to profit from an analysis mesh that is aligned with the shape parametrization in large part, we rotate the embedding domain $\Omega$ by 25 degrees counter-clockwise against $\Gamma^{h}$, cf. Fig. 11b. For a first visual inspection of the characteristics for the embedding domain discretization technique, we show in Fig. 12 a comparison of the strain energy density distribution that is obtained for a standard (conforming) finite element analysis mesh on the one hand, and the reorientation scheme on the other hand. Both contour plots are scaled to the same relative $(0-1)$ range of values, and we observe a qualitatively similar distribution of the strain energy density.

In a first optimization run, we preclude the influence of the regularization vector $\mathbf{r}$ on the combined descent direction by setting the angular threshold value $\eta=1$ in (53). Thereby, the steepest descent $\mathbf{d}$ is employed as a search direction in each design iteration. After very few iterations, and depending on the number of vertices $\mathbf{v}^{(k)}$ that we place alongside $\Gamma^{h}$, we cannot continue the design update procedure due to segment intersections among adjacent $\Gamma_{s}$, cf. Fig. 13a. In fact, only for the coarsest possible parametrization, in which four vertices $\mathbf{v}^{(k)}$ are used to vary $\Gamma^{V}$, we 
arrive at the sought-after optimal shape ${ }^{8}$.

Now, by lowering the angular threshold value $\eta$, and hence including the influence of the regularization vector $\mathbf{r}$ on the combined descent direction $\widehat{\mathbf{d}}$, we arrive at the sought-after optimal shape also when using higher-level parametrizations $\Gamma^{h}$. For the study depicted in Fig. 13b, we use $\eta=0.8$ for the angular threshold value. We allow for 100 design iterations in total which required a CPU time of 27 seconds to complete. A convergence history for the objective and constraint function is given in Figs. 14 and 15, respectively. We observe an increasing violation of the compliance constraint until design iteration 25. Through a repeated update of the Lagrange multiplier estimate and the penalty parameter via (60) the constraint is then enforced at later design iterations. The objective function settles after a reduction of 26 percent of the initial volume. We found that the higher the level of the parametrization, the more we have to lower the angular threshold value to avoid the intersection of segments $\Gamma_{s}$ at early design stages. This motivates the consideration of two basic mechanisms for the speed-up of convergence rates in the presented optimal shape design approach. First, one may consider an adaptive adjustment of the angular threshold value $\eta$ for the course of the optimization. This adaptation process may for instance be stimulated by vertex-wise curvature measures, or the current decrease in the augmented objective value in (58). Specifically, this would provide a tool to facilitate the attainment of the optimal shape design at later design iterations in which the regularization movement of vertices does not alter the current shape design, cf. Fig. 13b.

Second, one may consider a staggered solution procedure in which one increases the level of the parametrization $\Gamma^{h}$ as soon as the decrease in the augmented objective function stalls. This may either take the form of a uniform refinement in which each segment $\Gamma_{s}$ is bisected, or a more adaptive approach, in which segments are marked for refinement based on local sensitivity or curvature measures.

The accompanying analysis mesh for the optimal shape design depicted in Fig. 13b is shown in Fig. 16a along with the distribution of the displacement component in the $x$-direction. Therein, the physical domain boundary is represented by both, boundary-conforming elements and elements that are intersected diagonally by $\Gamma^{h}$. This is due to the counter-clockwise rotation of $\Omega^{h}$ against $\Gamma^{h}$ for the initial guess in Fig. 11b. In fact, if we set this rotation aside and allow for another optimization run, the reorientation scheme renders an all conforming analysis mesh for the optimal design trial, cf. Fig. 16b. Both contour plots in Fig. 16 show a linear increase of the displacement component in the $x$-direction from the constrained left-hand edge to the loaded right-hand edge. Also, we obtain vertical slides within the contour field, i.e. perpendicular to the direction of force application, as expected from the analytical one-dimensional considerations for a bar of uniform cross-section.

We obtain the same optimal shape design when using the staircase rather than the reorientation scheme for the embedding domain discretization. However, if we rely on the same thresholdvalue $\gamma$ for the adaptive mesh refinement within the boundary tracking procedure in (11), the domain update procedure tends to stall at later design iterations prior to the arrival at the desired optimal shape design. To circumvent this issue we either need to increase the number of integration points for the domain integral computations considering intersected elements, or sharpen the threshold value $\gamma$ for the tracking procedure. Both mechanisms lead to a higher computational effort of the staircase scheme as compared to the previously installed reorientation scheme.

\footnotetext{
${ }^{8}$ Even though this is only possible since the optimal shape design of a bar with uniform cross-section is within the accessible design space when using four vertices to represent $\Gamma^{V}$.
} 


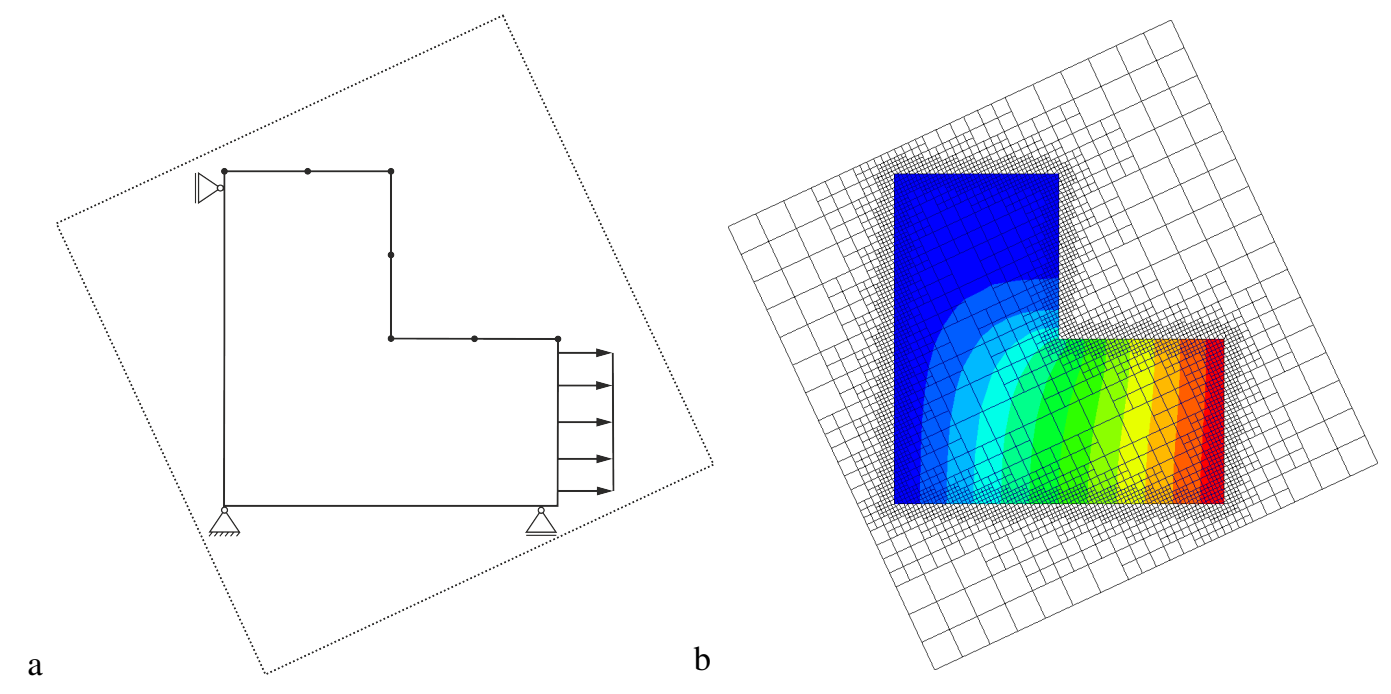

Figure 11: L-shape under uniaxial loading. a Setup of the mechanical problem: The bottom right-hand edge is subject to a uniform tension, whereas the left and bottom edge are constrained in the $x$ - and $y$-direction, respectively. The variable portion $\Gamma^{V}$ of the domain boundary for the course of the optimization is indicated by black marks. b An embedding domain discretization technique is employed for the solution of the mechanical problem. The contour plot shows the displacement component in the $x$-direction.

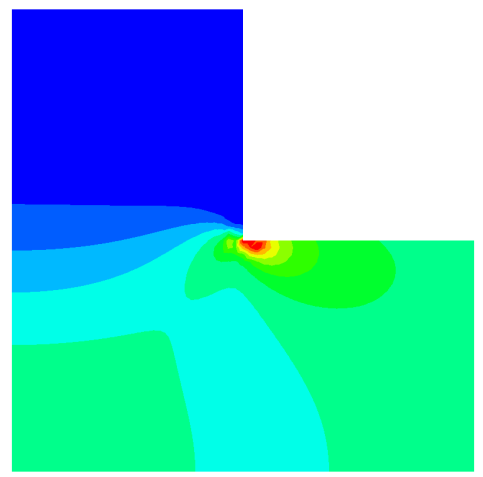

a



b

Figure 12: L-shape under uniaxial loading. Distribution of the strain energy density for the initial shape design. Both contour plots are scaled to the same relative $(0-1)$ range of values. a Reference solution obtained by a standard finite element method with conforming mesh. b Solution for the embedding domain discretization technique when using the reorientation scheme. For the visualization, we mask exterior elements. 


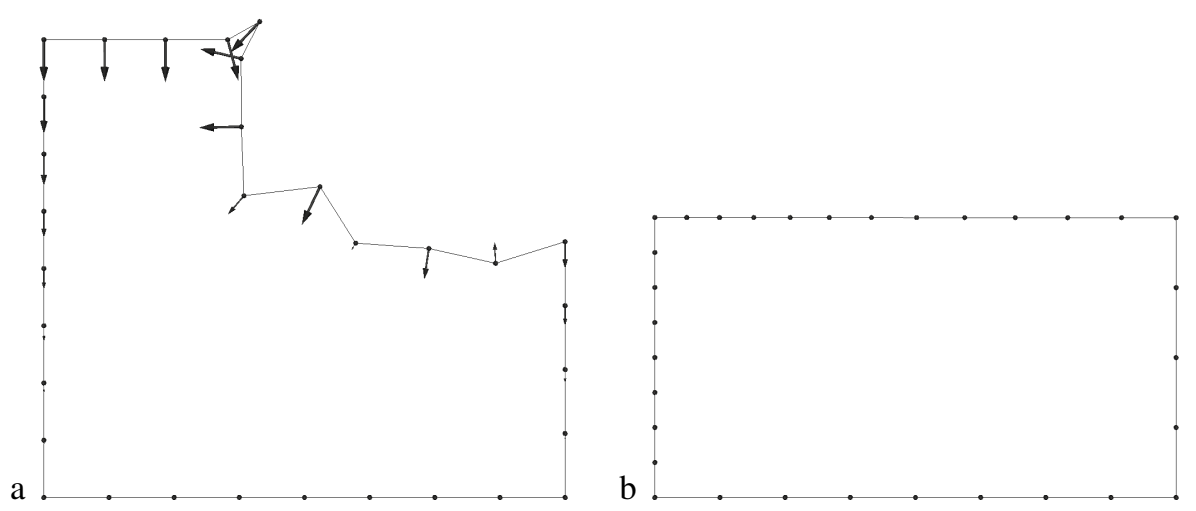

Figure 13: Influence of the regularization vector $\mathbf{r}$ on the course of the optimization. a When using the steepest descent direction d, no convergence is achieved for the L-shape optimal design problem. This is due to the highly non-regular boundary $\Gamma^{h}$ after very few design iterations that does not allow for a continuation of the domain update procedure. The vector plot shows the steepest descent direction for the current design trial. b Upon consideration of the regularization vector, and hence using the combined descent direction $\widehat{\mathbf{d}}$, the domain update procedure yields the desired optimal shape design.

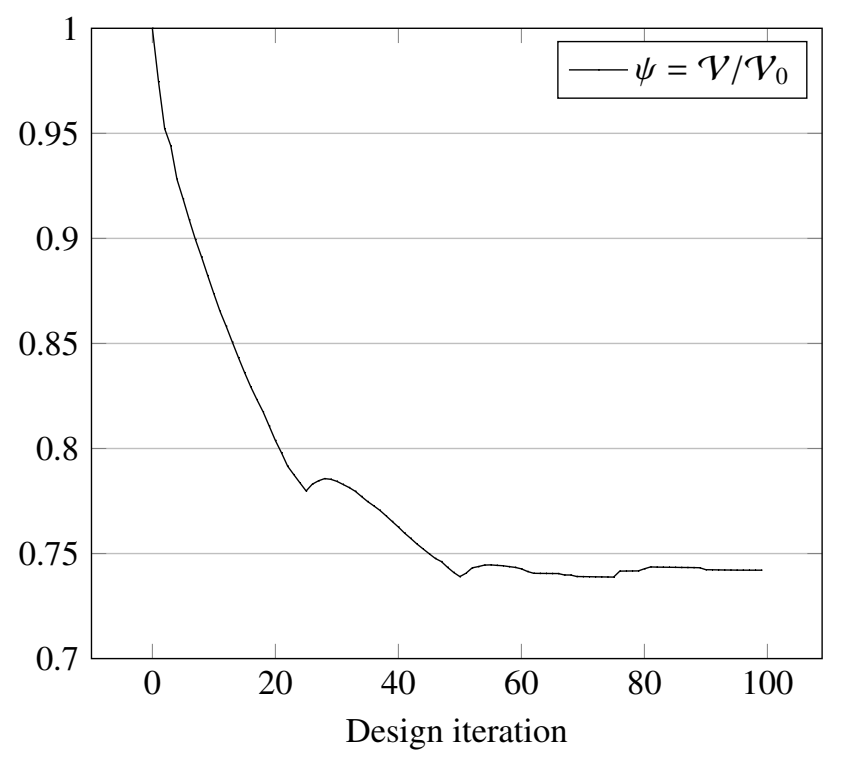

Figure 14: History of the volume objective function for the L-shape design problem. The volume is sharply reduced at early design iterations and settles after a reduction of 26 percent as compared to the initial design. 


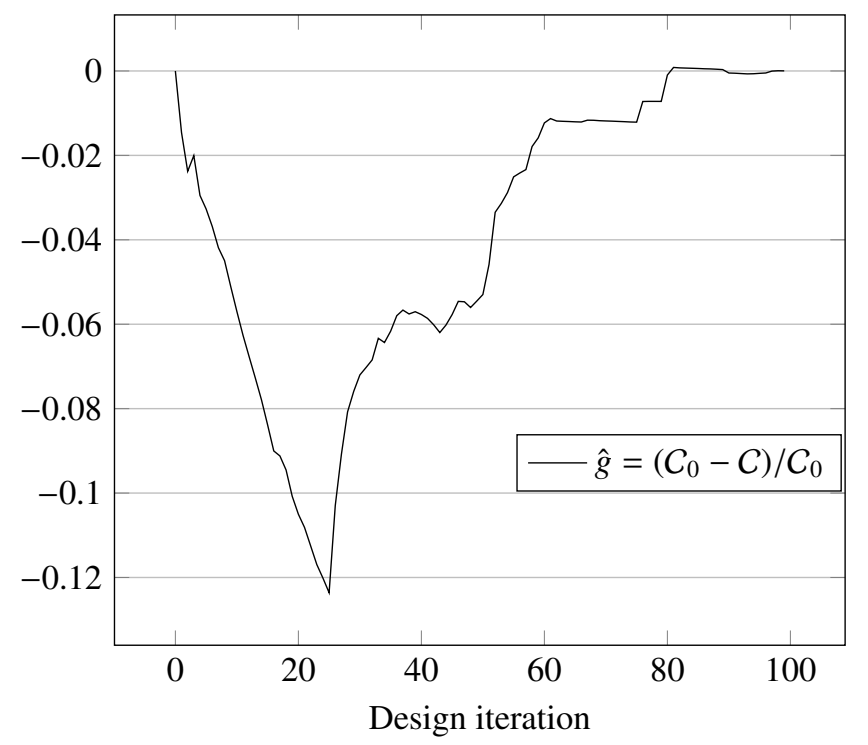

Figure 15: History of the compliance constraint function for the L-shape design problem. The constraint is increasingly violated until design iteration 25. Due to the update of the Lagrange multiplier estimate and the penalty parameter throughout the course of optimization, the constraint is then enforced at later design iterations.

a

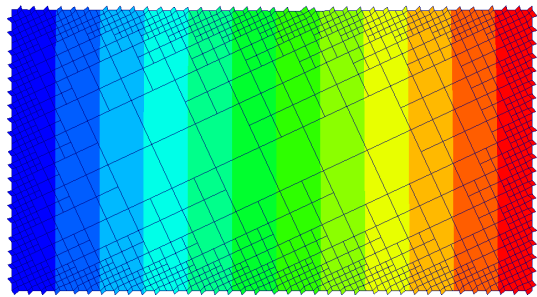

b
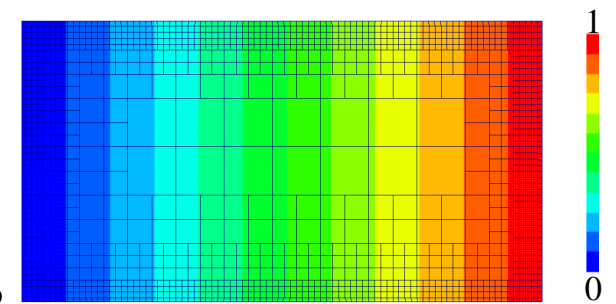

Figure 16: Distribution of the displacement component in the $x$-direction for the optimal shape design and using the reorientation scheme for the solution of the physical state problem within the embedding domain $\Omega$. Both contour plots are scaled to the same relative $(0-1)$ range of values and we mask exterior elements for ease of visualization. a Analysis mesh for the case in which the embedding domain is rotated by 25 degrees counter-clockwise against the initial guess $\Gamma^{h}$. The domain boundary $\Gamma^{h}$ is represented by both, a set of conforming elements and a set of elements that are intersected diagonally by $\Gamma^{h}$. b When using an embedding domain that is not rotated against the initial guess $\Gamma^{h}$, we obtain an analysis mesh that comprises conforming elements only. 


\subsection{Hook profile under uniaxial loading}

As a second example, we consider the hook profile depicted in Fig. 17a. The profile is constrained at the top edge, and subject to a pressure-type loading in the lower half of the circular cut-out. This loading may for instance be introduced through a fitted bolt, which is then pulled in the negative $y$-direction. The aim of this study is to minimize the hook volume $\mathcal{V}$, while keeping the compliance $C$ constant. The partitioning of the initial design layout into a variable boundary portion $\Gamma^{V}$ and a non-variable boundary portion $\Gamma^{P}$ is depicted in Fig. 17b.

For the solution of the physical state problem throughout the course of the optimization, we rely on the staircase discretization scheme, where we provide 25 integration points for the selective domain integration considering elements intersected by $\Gamma^{h}$. Further, we consider a staggered solution procedure in which we refine uniformly the domain parametrization $\Gamma^{h}$ after a predefined number of design iterations. This mechanism is accompanied by sharpening the threshold value $\gamma$ for the adaptive mesh refinement within the domain tracking procedure in (11).

After two such refinements in the level of the parametrization $\Gamma^{h}$, and a total number of 300 design iterations, we arrive at the shape design depicted in Fig. 18. The accompanying contour plot shows the distribution of the strain energy density for the initial and the final design layout, respectively. We observe that regions that show low strain energy densities and hence do not contribute a large share to the loading capacity of the structure are shrinked, whereas regions of high strain energy density tend to grow. In other words, a more efficient usage of the available material is accomplished. A selection of different stages in the evolution of the shape parametrization $\Gamma^{h}$ is shown in Figs. 19a-d. For the optimal design layout in Fig. 19d, we attain a volume reduction by 44 percent and observe the same compliance as for the initial design layout in Fig. 19a. The CPU time to complete the 300 design iterations was 339 seconds.

We obtain a similar result for this numerical study when employing the reorientation rather than the staircase scheme for the solution of the physical state problem throughout the course of the optimization. However, we find that for high-level parametrizations $\Gamma^{h}$, the reorientation scheme tends to render elements of poor quality in regions of $\Gamma^{h}$ that show acute angles between adjacent segments $\Gamma_{s}$. One can partly account for this drawback by lowering the threshold-value $\gamma$ for the adaptive mesh refinement within the boundary tracking procedure, so as to keep the characteristic edge length in $\{\Omega\}^{\text {bnd }}$ well below the size of $\Gamma_{s}$ in the respective region. Further, one may counteract the formation of acute angles during the course of the optimization by lowering the sufficient decrease condition $\eta$, and hence emphasizing the influence of the regularization vector on the domain update procedure at early design stages.

The first mechanism leads to a higher computational cost for every single design iteration, whereas the second mechanism requires a greater total number of design iterations to reach the optimal design layout. Still, both mechanism may not be adequate to avoid local mesh entanglement in regions that feature acute angles in the optimal design layout, as is the case for the tip of the evolving hook design in Fig 19d. To this extent, we formulate a minimum angle constraint for the respective region of interest, which was checked after every design iteration.

\subsection{Cantilever beam under bending load}

For a final numerical study, we consider the cantilever beam depicted in Fig. 20. The beam contains four rectangular cut-outs and is subject to a centred distributed load at the right-hand edge, whereas the left-hand edge is constrained. We aim to minimize the volume $\mathcal{V}$ of the structure, while keeping its compliance $C$ at a constant level. The solution of the physical boundary problem is obtained by use of the staircase discretization technique. For the course of the optimization, we consider a bounding box constraint, such that vertices $\mathbf{v}^{(k)}$ cannot go beyond the 

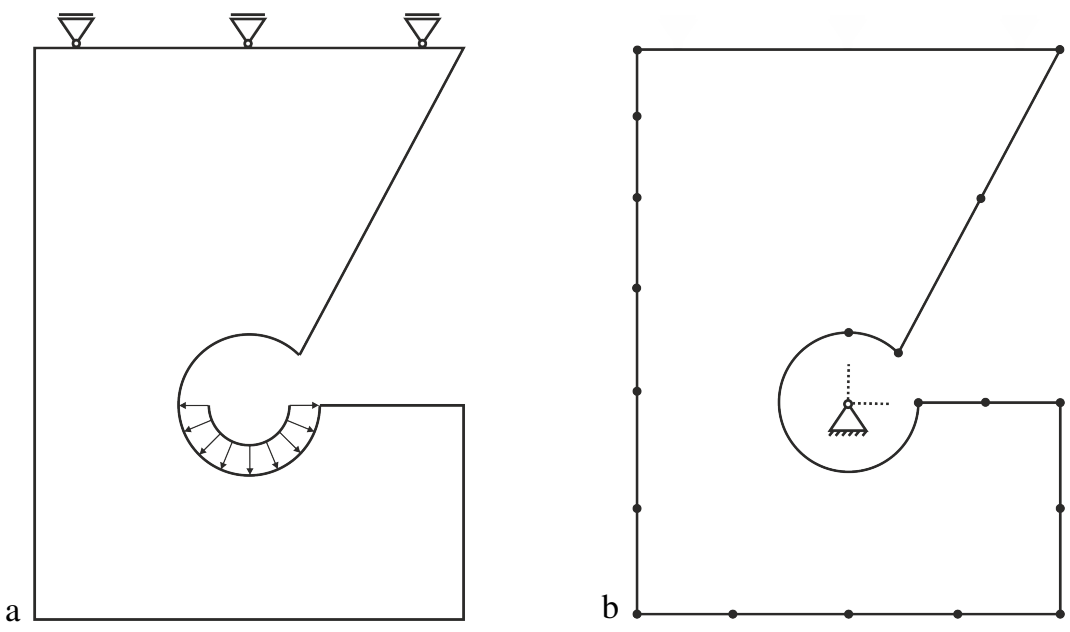

Figure 17: Hook profile under uniaxial loading. a Setup of the mechanical problem. The top horizontal edge is constrained, whereas the lower half of the circular cut-out is subject to pressure-like loading. This loading may for instance be introduced through a fitted bolt which is then pulled in the negative $y$-direction. b Subdivision of the domain boundary $\Gamma^{h}$ into a variable portion $\Gamma^{V}$ (indicated by black marks) and a non-variable portion $\Gamma^{P}$ for the course of the optimization.
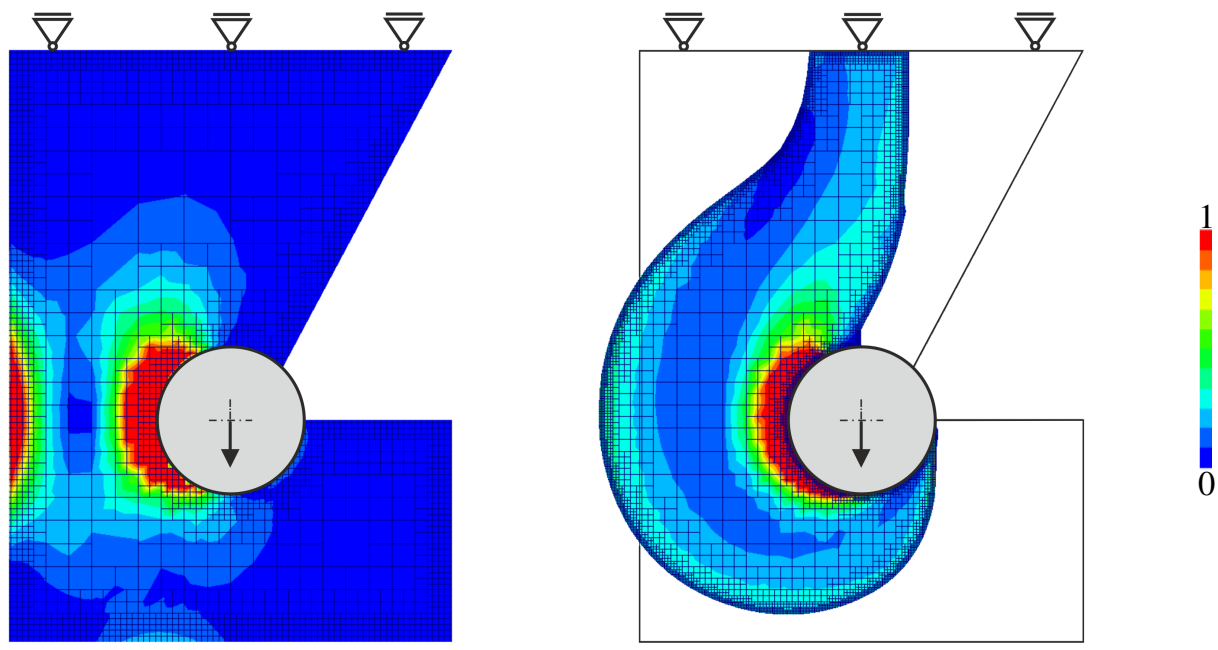

Figure 18: Hook profile under uniaxial loading. Distribution of the strain energy density for the initial and the optimal design layout, respectively. Both contour plots are scaled to the same relative $(0-1)$ range of values and we mask exterior elements for ease of visualization. 
a
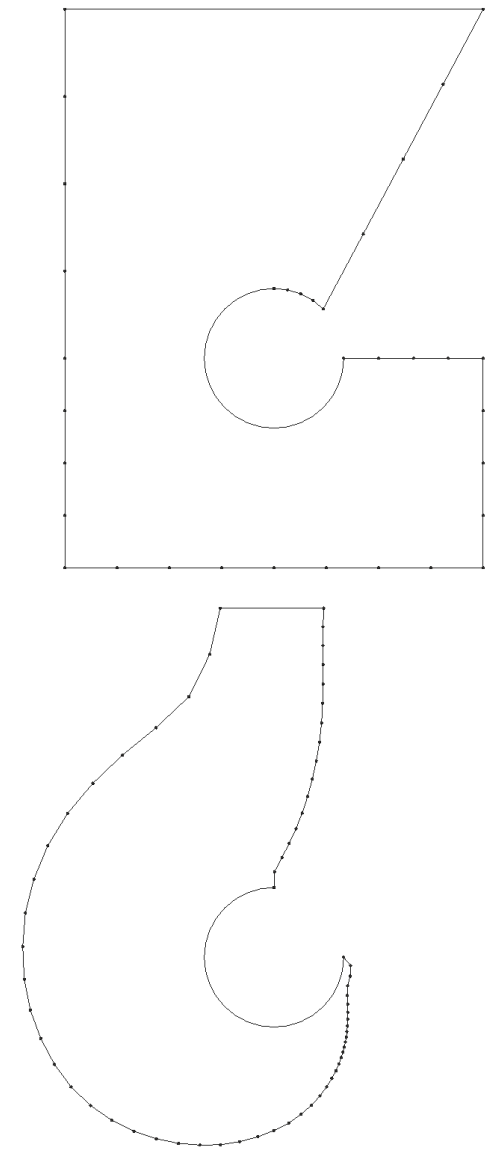

Figure 19: Hook profile under uniaxial loading. Evolution of the shape parametrization $\Gamma^{h}$ throughout the course of the optimization. a Initial design layout with low-level parametrization $\Gamma^{h, 0}$. b Intermediate design trial after 99 design iterations and still using the low-level parametrization $\Gamma^{h, 0}$. $\mathbf{c}$ Intermediate design trial after 199 design iterations and using the level one parametrization $\Gamma^{h, 1}$ that is obtained for a uniform refinement of $\Gamma^{h, 0}$. d Optimal design layout arrived at after 299 design iterations and using the level two parametrization $\Gamma^{h, 2}$. 
outline of the initial design layout. Further, the area of force application at the right-hand edge is held fixed for the optimization.

Again, we employ a staggered optimization routine in which we increase the level of the parametrization after completion of a fixed number of design iterations. Specifically, we allow for a total number of 300 design iterations, where we refine uniformly the parametrization $\Gamma^{h}$ after every 100 design iterations. Also, we sharpen the angular threshold value $\eta$ for the sufficient decrease condition after every 50 design iterations in order to lessen the influence of the regularization vector $\mathbf{r}$ on the combined descent direction $\widehat{\mathbf{d}}$. This is to speed-up convergence towards the optimal design layout at later design iterations. In the present context, this works against the tendency of the regularization vector to shrink the internal cut-outs.

In Fig. 21, we show the distribution of the strain energy density for the initial and the optimal design layout arrived at after 300 design iterations, respectively. The required CPU time for this numerical study was 259 seconds. A selection of different stages for the design evolution process is given in Figs. 22a-d. We observe the formation of a triangular-shaped cut-out at the left-hand edge, and a reorientation of the internal cut-outs to form a truss-like internal layout. For this optimal design layout depicted in Fig. 22d, we attain a volume reduction by 27 percent, and observe the same compliance as for the initial design layout in Fig. 22a.

We obtain a similar result for this numerical study when employing the reorientation rather than the staircase scheme for the solution of the physical state problem throughout the course of the optimization. However, as for the hook design problem outlined above, we need to take the same precautions to not suffer from mesh entanglements in regions that show acute angles. These mechanisms result in a higher number of design iterations as well as a higher computational cost for every single design iteration. Albeit, as opposed to the hook design problem, no acute angles are present in the optimal design layout such that no minimum angle constraint needs to be specified.

To this extent, we conclude that the staircase embedding domain technique is a more suitable tool to assist the design evolution process as compared to the reorientation scheme. This is particularly true when using a comparatively high-level parametrization $\Gamma^{h}$ for the course of the optimization, as well as for the representation of large design changes. Furthermore, the extension of the present optimization framework to a three-dimensional setting is rather straightforward for the staircase scheme, but requires a much more involved course of action for the reorientation scheme. Still, in order to account for the lower accuracy when evaluating domain integrals for intersected elements in the staircase scheme, as it was observed for the introductory L-shape design problem, it seems promising to replace the integration point oversampling technique by an exact integration. This may be best accomplished by use of a separate intra-element sub-triangulation using triangular or tetrahedral elements, respectively.

\section{Summary and outlook}

We presented a method for structural shape optimization that relies on an embedding domain discretization technique. Using an explicit boundary description for the course of the optimization, the embedding domain is subdivided into interior elements, exterior elements, and elements intersected by the evolving shape design.

We consider two different techniques to take into account the geometric mismatch between the structural component and its embedding domain representation. First, in the staircase scheme, a selective domain integration for intersected elements is achieved by use of integration point 

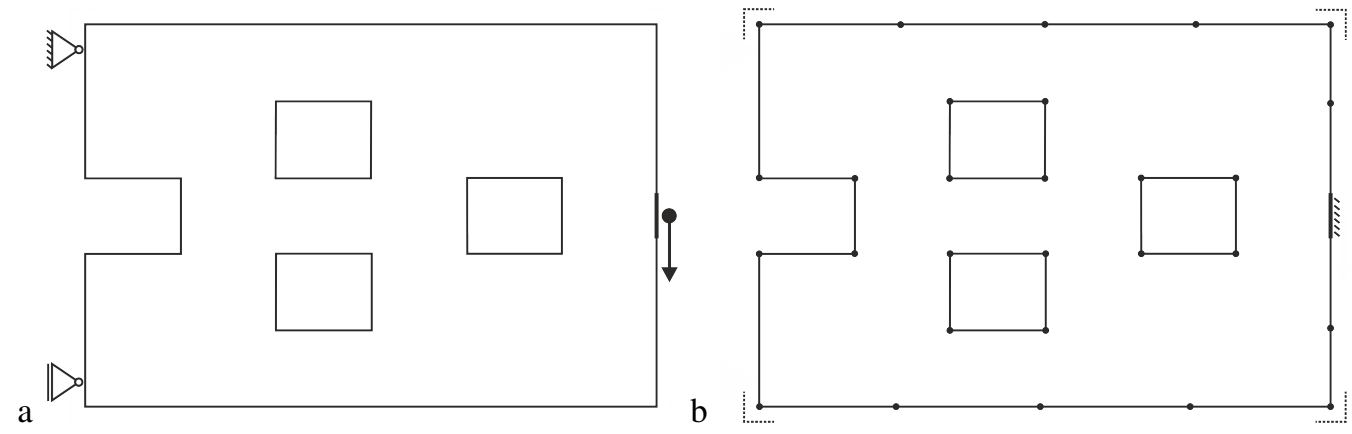

Figure 20: Cantilever beam under bending load. a Setup of the mechanical problem. The left edge is constrained, whereas the right edge is equipped with a centred distributed load acting in the negative $y$-direction. b Subdivision of the boundary into a variable and a non-variable portion for the course of the optimization. Also, we consider a bounding box constraint, such that vertices $\mathbf{v}^{(k)}$ cannot leave the outline of the initial design layout.
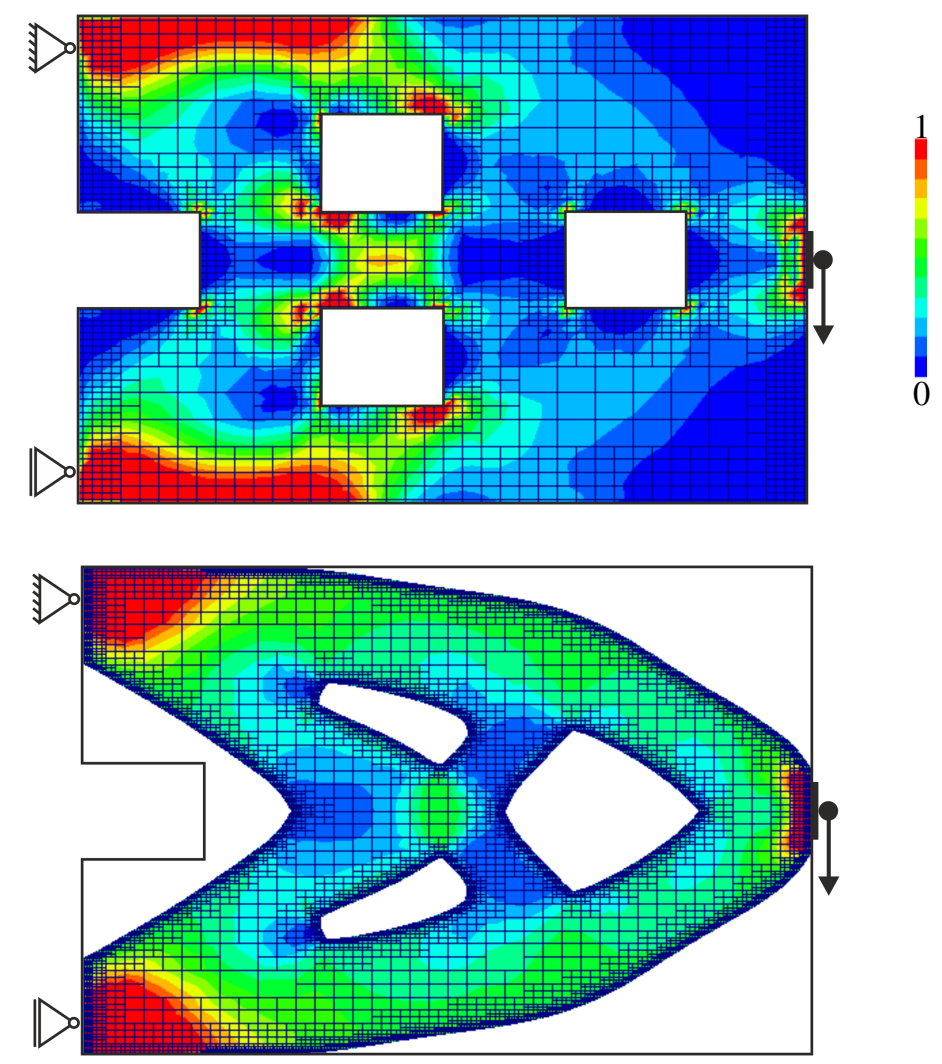

Figure 21: Cantilever beam under bending load. Distribution of the strain energy density for the initial and the optimal design layout, respectively. Both contour plots are scaled to the same relative $(0-1)$ range of values and we mask exterior elements for ease of visualization. 

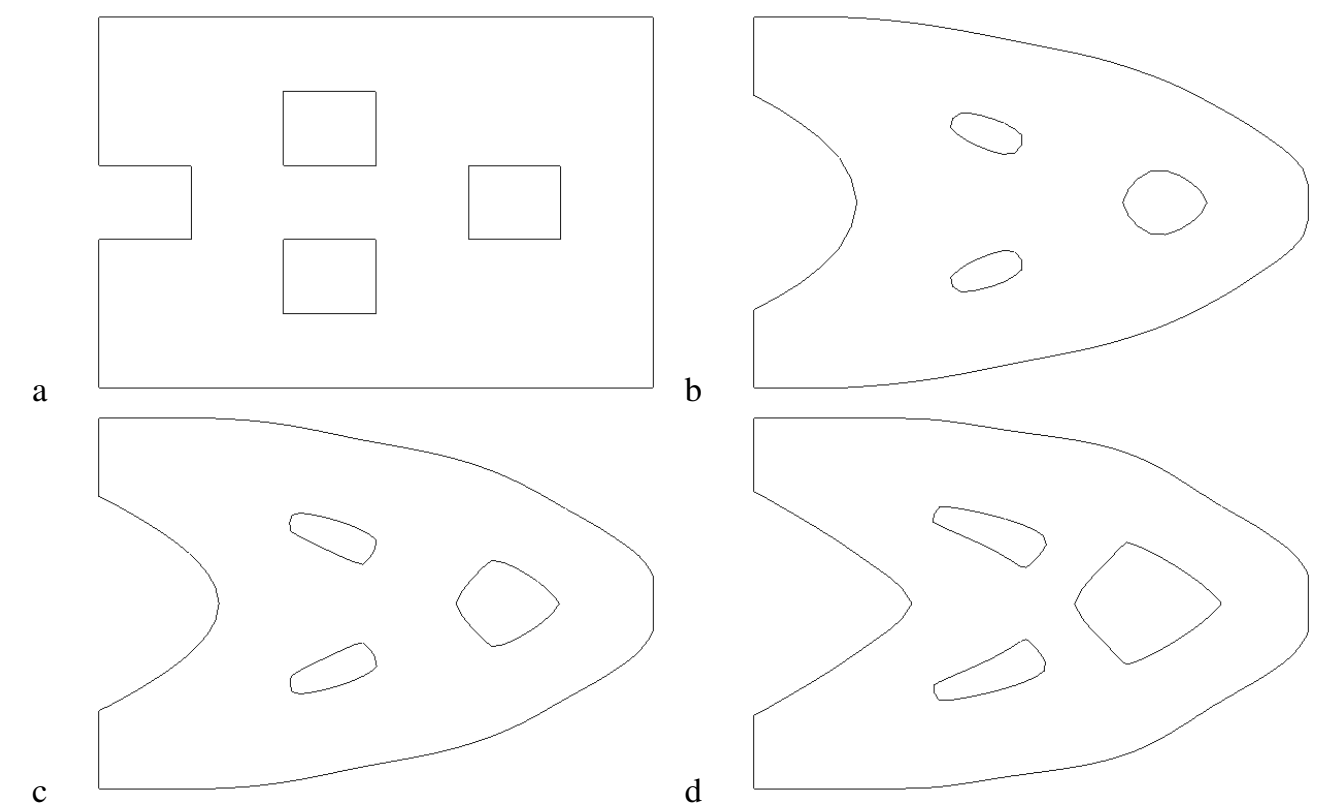

Figure 22: Cantilever beam under bending load. Evolution of the parametrization $\Gamma^{h}$ throughout the course of the optimization. a Initial design layout. b/c Design layout after 99 and 199 design iterations, respectively. d Optimal design layout arrived at after 299 design iterations.

oversampling. Thereby, every integration point is checked to be interior or exterior to the physical domain boundary, and hence its respective integral contribution is either taken into account or discarded for the evaluation of the domain integral. On the contrary, using the reorientation scheme, we aim to reorient intersected elements such as to obtain a layer of conforming elements within the otherwise uniform embedding domain discretization. This enables the use of a standard numerical integration procedure for the domain integration of the evolving shape design.

By use of three numerical examples, we found the staircase scheme to be a more suitable tool to accompany the design evolution process in the two-dimensional setting. Further, its extension to a three-dimensional setting seems straightforward, whereas the reorientation scheme requires a complete redefinition of the necessary course of action. In order to enhance the accuracy of the selective domain integration procedure within the staircase scheme, we reserve the use of a separate intra-element sub-triangulation for future investigations. Also, it seems promising to augment the presented shape optimization framework with a topological sensitivity analysis to trigger topological changes throughout the course of the optimization $[66,67,68,69]$.

As an overall impression, we found that the solution of shape optimal design problems using an embedding domain discretization technique is advantageous as compared to a classical Lagrangian approach when it comes to the representation of large design changes. Due to the embedding domain setting, one avoids the need to provide a conforming finite element mesh at every design iteration, and hence circumvents costly mesh smoothing or re-meshing operations. Still, through a direct manipulation of boundary vertices for the domain updates, we preserve an essential characteristic of the Lagrangian approach as we do not limit the admissible design space for the course of the optimization. Thereby, the adaptive geometric regularization tech- 
nique in use proved effective in avoiding irregular design updates at early design stages, and still providing feasible descent directions for the course of the optimization.

\section{References}

[1] V. Braibant, C. Fleury, Shape optimal design using B-splines, Computer Methods in Applied Mechanics and Engineering 44 (1984) 247 - 267

[2] R. T. Haftka, R. V. Grandhi, Structural shape optimization: A survey, Computer Methods in Applied Mechanics and Engineering 57 (1986) 91 - 106.

[3] Y. Ding, Shape optimization of structures: a literature survey, Computers \& Structures 24 (1986) 985 - 1004

[4] J. Bennett, M. Botkin, Structural shape optimization with geometric description and adaptive mesh refinement, AIAA journal 23 (1985) 458-464.

[5] K.-H. Chang, K. K. Choi, A geometry-based parameterization method for shape design of elastic solids, Mechanics of Structures and Machines 20 (1992) 215 - 252.

[6] A. Falk, F. J. Barthold, E. Stein, A hierarchical design concept for shape optimization based on the interaction of CAGD and FEM, Structural Optimization 18 (1999) 12 - 23.

[7] W. A. Wall, M. A. Frenzel, C. Cyron, Isogeometric structural shape optimization, Computer Methods in Applied Mechanics and Engineering 197 (2008) 2976 - 2988.

[8] N. Kikuchi, K. Chung, T. Torigaki, J. Taylor, Adaptive finite element methods for shape optimization of linearly elastic structures, Computer Methods in Applied Mechanics and Engineering 57 (1986) 67 - 89.

[9] T.-M. Yao, K. K. Choi, 3-d shape optimal design and automatic finite element regridding, International Journal for Numerical Methods in Engineering 28 (1989) 369 - 384.

[10] P. Morin, R. H. Nochetto, M. S. Pauletti, M. Verani, Adaptive finite element method for shape optimization, ESAIM: COCV 18 (2012) 1122 - 1149

[11] S. Riehl, P. Steinmann, An integrated approach to shape optimization and mesh adaptivity based on material residual forces, Computer Methods in Applied Mechanics and Engineering 278 (2014) 640 - 663.

[12] E. Nadal, J. J. Ródenas, J. Albelda, M. Tur, J. E. Tarancón, F. J. Fuenmayor, Efficient finite element methodology based on cartesian grids: Application to structural shape optimization, Abstract and Applied Analysis 2013 (2013).

[13] N. H. Kim, Y. Chang, Eulerian shape design sensitivity analysis and optimization with a fixed grid, Computer Methods in Applied Mechanics and Engineering 194 (2005) 3291 - 3314.

[14] A. R. Najafi, M. Safdari, D. A. Tortorelli, P. H. Geubelle, A gradient-based shape optimization scheme using an interface-enriched generalized FEM, Computer Methods in Applied Mechanics and Engineering 296 (2015) 1 17.

[15] J. Sethian, A. Wiegmann, Structural boundary design via level set and immersed interface methods, Journal of Computational Physics 163 (2000) 489 - 528.

[16] P. Duysinx, L. Van Miegroet, T. Jacobs, C. Fleury, Generalized shape optimization using X-FEM and level set methods, in: IUTAM Symposium on Topological Design Optimization of Structures, Machines and Materials Solid Mechanics and Its Applications, Springer Netherlands, 2006, pp. 23-32.

[17] S. Yamasaki, T. Nomura, A. Kawamoto, K. Sato, S. Nishiwaki, A level set-based topology optimization method targeting metallic waveguide design problems, Int. J. Numer. Meth. Engng. 87 (2011) 844 - 868.

[18] M. P. Bendsoe, O. Sigmund, Material interpolation schemes in topology optimization, Archive of Applied Mechanics 69 (1999) 635-654.

[19] G. Allaire, F. Jouve, A.-M. Toader, Structural optimization using sensitivity analysis and a level-set method, Journal of Computational Physics 194 (2004) 363 - 393.

[20] M. García-Ruíz, G. Steven, Fixed grid finite elements in elasticity problems, Engineering Computations 16 (1999) $145-164$.

[21] P. D. Dunning, H. A. Kim, G. Mullineux, Investigation and improvement of sensitivity computation using the areafraction weighted fixed grid FEM and structural optimization, Finite Elements in Analysis and Design 47 (2011) $933-941$.

[22] F. Bobaru, S. Rachakonda, E(FG)2: a new fixed-grid shape optimization method based on the element-free galerkin mesh-free analysis, Structural and Multidisciplinary Optimization 32 (2006) 215-228.

[23] J. Parvizian, A. Düster, E. Rank, Finite cell method, Computational Mechanics 41 (2007) 121-133.

[24] A. Düster, J. Parvizian, Z. Yang, E. Rank, The finite cell method for three-dimensional problems of solid mechanics, Computer Methods in Applied Mechanics and Engineering 197 (2008) 3768 - 3782.

[25] J. Parvizian, A. Düster, E. Rank, Topology optimization using the finite cell method, Optimization and Engineering 13 (2012) 57-78. 
[26] R. van Loon, P. D. Anderson, J. de Hart, F. P. T. Baaijens, A combined fictitious domain/adaptive meshing method for fluid-structure interaction in heart valves, International Journal for Numerical Methods in Fluids 46 (2004) 533 -544 .

[27] R. Rangarajan, A. J. Lew, Universal meshes: A method for triangulating planar curved domains immersed in nonconforming meshes, Int. J. Numer. Meth. Engng 98 (2014) 236 - 264.

[28] G. Allaire, C. Dapogny, P. Frey, Topology and geometry optimization of elastic structures by exact deformation of simplicial mesh, Comptes Rendus Mathematique 349 (2011) 999 - 1003.

[29] G. Allaire, C. Dapogny, P. Frey, Shape optimization with a level set based mesh evolution method, Computer Methods in Applied Mechanics and Engineering 282 (2014) 22 - 53.

[30] S.-H. Ha, S. Cho, Level set based topological shape optimization of geometrically nonlinear structures using unstructured mesh, Computers \& Structures 86 (2008) 1447 - 1455.

[31] J. Bishop, Rapid stress analysis of geometrically complex domains using implicit meshing, Computational Mechanics 30 (2003) $460-478$.

[32] O. Schmitt, J. Friederich, S. Riehl, P. Steinmann, On the formulation and implementation of geometric and manufacturing constraints in node-based shape optimization, Structural and Multidisciplinary Optimization 53 (2016) 881-892.

[33] A. N. Christiansen, M. Nobel-Jørgensen, N. Aage, O. Sigmund, J. A. Bærentzen, Topology optimization using an explicit interface representation, Structural and Multidisciplinary Optimization 49 (2014) 387-399.

[34] A. N. Christiansen, J. A. Bærentzen, M. Nobel-Jørgensen, N. Aage, O. Sigmund, Combined shape and topology optimization of 3d structures, Computers \& Graphics 46 (2015) 25 - 35

[35] T. J. Hughes, The finite element method: linear static and dynamic finite element analysis, Courier Corporation, 2012.

[36] T. Zhu, S. Atluri, A modified collocation method and a penalty formulation for enforcing the essential boundary conditions in the element free galerkin method, Computational Mechanics 21 (1998) 211-222.

[37] T. Belytschko, Y. Y. Lu, L. Gu, Element-free galerkin methods, International Journal for Numerical Methods in Engineering 37 (1994) 229-256.

[38] S. Fernández-Méndez, A. Huerta, Imposing essential boundary conditions in mesh-free methods, Computer methods in applied mechanics and engineering 193 (2004) 1257-1275.

[39] D. Schillinger, M. Ruess, The finite cell method: A review in the context of higher-order structural analysis of CAD and image-based geometric models, Arch Computat Methods Eng (2014) 1 - 65.

[40] P. M. Knupp, Achieving finite element mesh quality via optimization of the Jacobian matrix norm and associated quantities. Part I:a framework for surface mesh optimization, International Journal for Numerical Methods in Engineering 48 (2000) $401-420$.

[41] H. Rathod, K. Nagaraja, B. Venkatesudu, N. Ramesh, Gauss legendre quadrature over a triangle, Journal of the Indian Institute of Science 84 (2004) 183-188.

[42] D. A. Dunavant, High degree efficient symmetrical gaussian quadrature rules for the triangle, International Journal for Numerical Methods in Engineering 21 (1985) 1129-1148.

[43] K. K. Choi, E. J. Haug, Shape design sensitivity analysis of elastic structures, Journal of Structural Mechanics 11 (1983) $231-269$.

[44] K. K. Choi, K.-H. Chang, A study of design velocity field computation for shape optimal design, Finite Elements in Analysis and Design 15 (1994) 317 - 341.

[45] F. Murat, J. Simon, Sur le contrôle par un domain géométrique, Technical Report RR-76015, Laboratoire d'Analyse Numerique, 1976.

[46] J. Céa, Conception optimale ou identification de formes, calcul rapide de la dérivée directionnelle de la fonction coût, RAIRO-Modélisation mathématique et analyse numérique 20 (1986) 371-402.

[47] J. S. Arora, J. B. Cardoso, Variational principle for shape design sensitivity analysis, AIAA Journal 30 (1992) 538 $-547$.

[48] J. S. Arora, An exposition of the material derivative approach for structural shape sensitivity analysis, Computer Methods in Applied Mechanics and Engineering 105 (1993) 41 - 62.

[49] J. Sokolowski, J. Zolésio, Introduction to shape optimization: Shape sensitivity analysis, Springer Ser. Comput. Math 10 (1992).

[50] M. Firl, R. Wüchner, K.-U. Bletzinger, Regularization of shape optimization problems using FE-based parametrization, Structural and Multidisciplinary Optimization 47 (2013) 507 - 521.

[51] N. Gerzen, D. Materna, F.-J. Barthold, The inner structure of sensitivities in nodal based shape optimisation, Computational Mechanics 49 (2012) 379 - 396

[52] H. Azegami, K. Takeuchi, A smoothing method for shape optimization: traction method using the robin condition, International Journal of Computational Methods 3 (2006) 21 - 33

[53] S. Riehl, J. Friederich, M. Scherer, R. Meske, P. Steinmann, On the discrete variant of the traction method in parameter-free shape optimization, Computer Methods in Applied Mechanics and Engineering 278 (2014) 119 - 
144.

[54] C. Le, T. Bruns, D. Tortorelli, A gradient-based, parameter-free approach to shape optimization, Computer Methods in Applied Mechanics and Engineering 200 (2011) 985 - 996.

[55] Y. Ohtake, A. Belyaev, I. Bogaevski, Mesh regularization and adaptive smoothing, Computer-Aided Design 33 (2001) $789-800$.

[56] D. G. Luenberger, Y. Ye, Linear and nonlinear programming, Springer, 2008

[57] J. Nocedal, S. Wright, Numerical Optimization, Springer Series in Operations Research Series, Springer-Verlag $\mathrm{GmbH}, 1999$.

[58] O. Pironneau, Optimal shape design for elliptic systems, Springer, 1984.

[59] J. Herskovits, G. Santos, On the computer implementation of feasible direction interior point algorithms for nonlinear optimization, Structural optimization 14 (1997) 165 - 172.

[60] J. Herskovits, P. Mappa, E. Goulart, C. M. Soares, Mathematical programming models and algorithms for engineering design optimization, Computer Methods in Applied Mechanics and Engineering 194 (2005) 3244 3268.

[61] R. Rockafellar, The multiplier method of Hestenes and Powell applied to convex programming, Journal of Optimization Theory and Applications 12 (1973) 555-562.

[62] M. Avriel, Nonlinear Programming: Analysis and Methods, Dover Books on Computer Science Series, Dover Publications, 2003.

[63] W. Bangerth, D. Davydov, T. Heister, L. Heltai, G. Kanschat, M. Kronbichler, M. Maier, B. Turcksin, D. Wells, The deal. II library, version 8.4, Journal of Numerical Mathematics 24 (2016).

[64] W. Bangerth, T. Heister, L. Heltai, G. Kanschat, M. Kronbichler, M. Maier, B. Turcksin, T. D. Young, The deal . ii library, version 8.1, arXiv preprint http://arxiv.org/abs/1312.2266v4 (2013).

[65] W. Bangerth, R. Hartmann, G. Kanschat, deal.II - a general purpose object oriented finite element library, ACM Trans. Math. Softw. 33 (2007) 24/1-24/27.

[66] S. Garreau, P. Guillaume, M. Masmoudi, The topological asymptotic for PDE systems: The elasticity case, SIAM J. Control Optim. 39 (2001) $1756-1778$

[67] A. Novotny, R. Feijóo, E. Taroco, C. Padra, Topological sensitivity analysis for three-dimensional linear elasticity problem, Computer Methods in Applied Mechanics and Engineering 196 (2007) 4354 - 4364.

[68] A. A. Novotny, J. Sokołowski, Topological derivatives in shape optimization, Springer Science \& Business Media, 2012.

[69] S. Riehl, P. Steinmann, A staggered approach to shape and topology optimization using the traction method and an evolutionary-type advancing front algorithm, Computer Methods in Applied Mechanics and Engineering 287 (2015) $1-30$ 